\title{
Some New and Previous Materials on Alpine Magmatism, Tectonics, Melt and Fluid Inclusions, and Metallogeny in Eastern Iran
}

\author{
A. Romanko $^{1}$, N.A. Imamverdiyev ${ }^{2}$, V. Prokofiev ${ }^{3}$, I. Vikentiev ${ }^{3}$, B. Rashidi ${ }^{4}$ \\ A. Savichev ${ }^{1}$, M. Heidari ${ }^{5}$ \\ ${ }^{1}$ Geological Institute, Academy of Sciences, Moscow, Russian Federation. \\ ${ }^{2}$ Baku State University, Baku, Republic of Azerbaijan. \\ ${ }^{3}$ Institute of geology of ore deposits, Academy of Sciences, Moscow, Russian Federation. \\ ${ }^{4}$ Satrap Resources, Perth, Australia. \\ ${ }^{5}$ Pars Kani, geoconsulting, Tehran, Iran.
}

*Corresponding Author: A. Romanko, Geological Institute, Academy of Sciences, Moscow, Russian Federation.Email: a.romanko@ya.ru,alx.romanko@gmail.com

\begin{abstract}
The Alpine tectonic-magmatic-metallogenic events in eastern Iran, Middle East and also in Lesser Caucasus - mainly Late Cenozoic (by N.A. Imamverdiyev et al.) have some common similarities. Important geological - metallogenic +- OIL / HC correlation for the Alpine time exists (metallogeny of East Iran led by outstanding regional trio: E. Romanko, A. Houshmandzadeh, and M.A.A. Nogole-Sadat). Geological northeastern (NE) zoning and "hot" tectonics due to the African superplume activity including, probably, known delamination of lithosphere during collision of mantle lithosphere ca. 13 Ma is principal here. Intraplate alkaline-subalkaline rocks of the region studied including Quaternary real carbonatites of Hanneshin, Afghanistan were derived from enriched African superplume-related mantle sources being enriched in HFSE - Nb, Ta, Zr, Y, P, Ti. Late Cenozoic High-K calc-alkaline rocks in the Lesser Caucasus could be deal with African superplume activity too despite their subduction-related rock geochemistry. Important data exist about a general meridianal-close (ca. N-S) zoning of oil / hydrocarbons (HC), muds, salts etc. here. This is one of arguments in favor of deep HC input alongside to traditional HC interpretation too. Large regional economic $\mathrm{Cu}$-Au porphyry etc. metallogeny deals mainly with Eocene (Pg2) shoshonite latite series rocks formed during subduction of Arabian plate beneath the Central Iran.
\end{abstract}

Keywords: eastern Iran, Middle East, conjunction in Alpine-Himalayan mobile belt, geology, geochemistry and petrology, tectonics, magmatism, metallogeny, African superPlume, delamination, mineralogy, melt and fluid inclusions, northeastern (NE) tectonic-magmatic-metallogenic +- oil / hydrocarbons (HC) zoning.

\section{INTRODUCTION}

The studied area is a small central fragment in the huge Alpine-Himalayan orogenic (collisional or mobile) superimport belt (Stocklin et al., 1965; Milanovsky, Koronovsky, 1973; Nogole-Sadat et al., 1985; Houshmandzadeh et al., 1986; E. Romanko et al, 1984; Khain, Leonov, 1988, Leonov et al, 2010; Trifonov, Ruzhentsev, 1984) and many other works. As we know, this fantastic region is very important. Geo-data in some structures of the region are poor. The first field materials were received by our team led by outstanding regional geo-trio as A. Houshmandzadeh, M.A.A. Nogole-Sadat and E. Romanko in fairly non-simple field conditions. Here are some new and previous data.

\section{OUTLINE OF GEOLOGY}

General geology and tectonics were characterized in such works as follows: (Nogole-Sadat, 1985; Houshmandzadeh et al., 1986; etc. and etc., fig. 1). Two groups of magmatic rocks were revealed here by our team as: mainly Eocene shoshonitic-latitic etc. rocks of the first group (subduction-related one) and principally other rocks - Neogene - Quaternary intraplate subalkaline and alkaline ones, second group (Romanko et al., 2005). 
Differentiated rocks of the first group are the products of a large mainly Paleogene subduction of the Arabian plate beneath the Central Iran block (Fig. 1). This subduction is confirmed by the regional tectonic analysis (Leonov et al., 2010), High-resolution tomography by known J. Ritsema's team (Bull et al., 2009 etc.), geochemistry (Romanko et al., 2013; etc., fig. 1) etc. Catastrophic earthquakes up to $8 \mathrm{M}$ and more, eunfortunately, are not rare here. A recent catastrophic precedent is 2003 Bam earthquake in East Iran with a lot of casualties. Strong seismic hazards in Pakistan, Agfghanistan, Turkey etc. including the very 2015 year bring civilian and economic damage.

Antipodes of the second group related to African superplume activity are: intraplate K-Na subalkaline and alkaline rocks - High-Ti trachybasalts, trachyandesites, real Quaternary carbonatites of Hanneshin, Afghanistan, Late Cenozoic carbonatites of Arabia, also Neogene lamproites of Algeria etc. by E. Romanko et al., 1988 and Romanko et al., 2013 (tables 1-11, fig. 1-2, 9-11; Bogatikov et al., 1987; Luchitsky, 1985; Shilov, 1997; Yarmolyuk et al., 2001; Knipper et al., 1992 etc.)

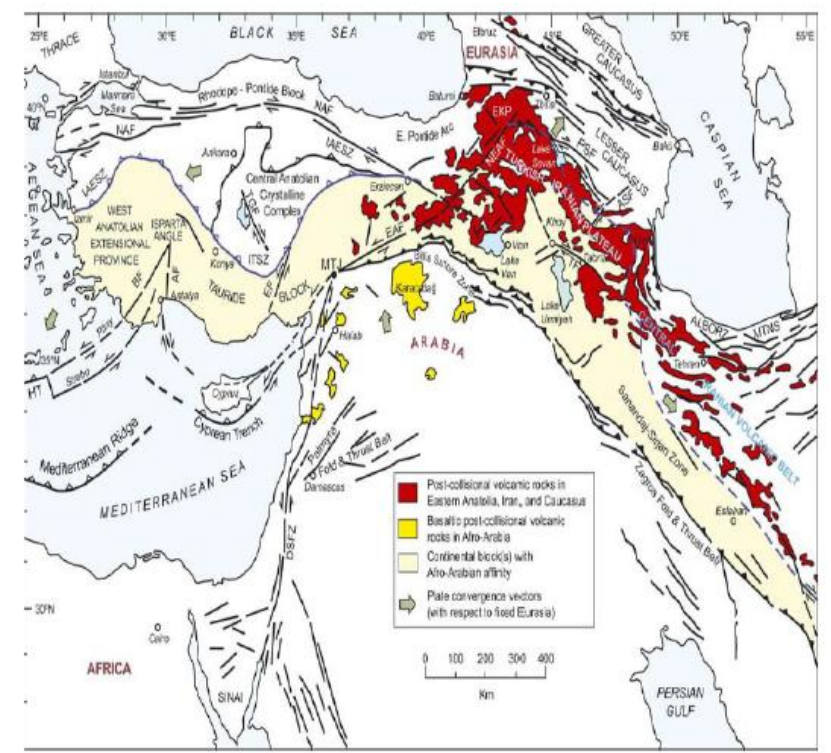

Fig1. Volcanic associations in the region studied, with special reference to the Lesser Caucasus using data by Dilek, Imamverdiyev, and Altunkaynak, 2010, with small change and simplification. Explanation (as in the very figure): 1 - red color - post-collisional differentiated volcanites including trachybasalts etc., 2 - yellow color mainly basalts in peri-Arabian region, 3 - continental blocks with Afro-Arabian affinity, 4 - plate convergence vectors with respect to fixed Eurasia.

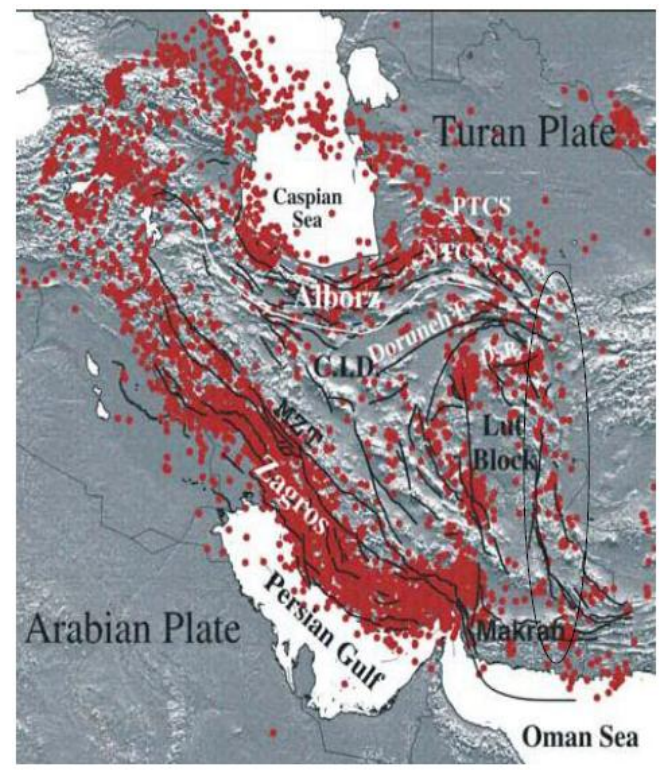

Fig1-2. Distribution of earthquake epicenters - red circles - in the Middle East after (Alinaghi et al., 2007). We can see known Lut Block and immediately next to the east - studied East Iran zone and adjacent structures marked by oval. 


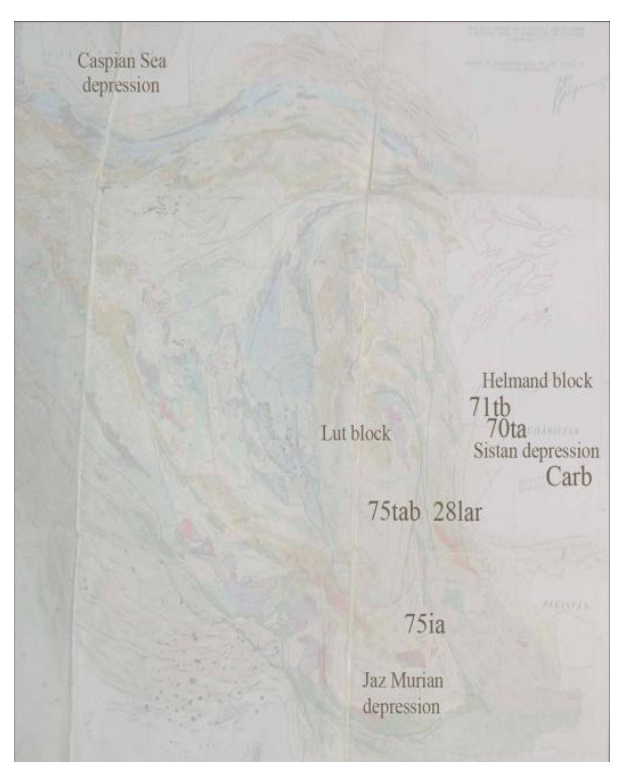

Fig1-3. Magmatic samples position in East Iran using Geological map scale 5: 000 000. R-70, R-71 - intraplate rocks in Sistan, R-75ia - High K-dacite with a high crystallization temperature as shown in text, Carb = carbonatites of Hanneshin, Afghanistan, R-28 - Lar alkaline intrusion with Cu-Au porphyry mineralization, Abbas Abad - important area with $C u$ deposits, tab = basic trachyandesite, $t b=$ trachybasalt.

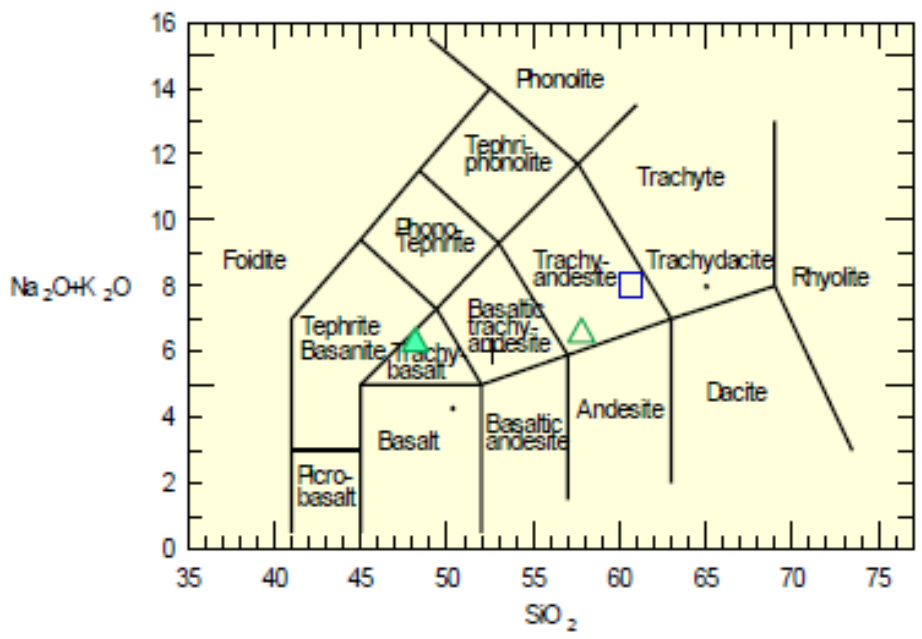

Fig2-1. $\mathrm{Na}_{2} \mathrm{O}+\mathrm{K}_{2} \mathrm{O}(w t \%)$ versus $\mathrm{SiO}_{2}(w t \%)$ or TAS diagram. Triangles - intraplate rocks of East Iran, $\mathrm{N}$ ? age, quadrangle - Lar alkaline intrusive massif with Cu-Au mineralization, Pg3? age, E. Iran/ W. Afghanistan border. Dot - trachydacite of shoshonite - latite series, Kurama zone, Tien-Shan, C3-P1, analogue of Pg2 shoshonite - latite series (Lesser Caucasus - Central Iran - East Iran).

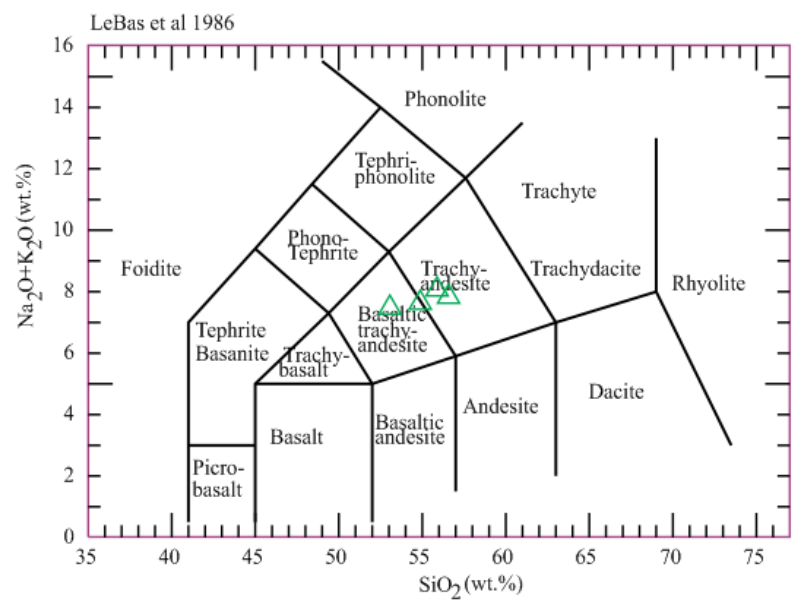

Fig2-2. $\mathrm{Na}_{2} \mathrm{O}+\mathrm{K}_{2} \mathrm{O}(w t \%)$ versus $\mathrm{SiO}_{2}(w t \%)$ or TAS diagram for the Abbas Abad Cu-mining area, Central Iran (NE Iran by a formal geography), Pg2? age. Samples of M. Heidari et al. 
Some New and Previous Materials on Alpine Magmatism, Tectonics, Melt and Fluid Inclusions, and Metallogeny in Eastern Iran

These intraplate rocks, in contrast to subduction-related calc-alkaline and other rocks, are characterized by an enrichment in both LILE - K, Rb, Ba, Cs and HFSE - Nb, Y, Ta, Zr, Ti, P, etc. (Tables or tab. 1, 5-11, fig. 3) with a characteristical positive $\mathrm{Eu} / \mathrm{Eu}^{*}$ - more than 1.0-1.1. Also, increased content of $\mathrm{P}_{2} \mathrm{O}_{5}-$ sometimes more than $1.0 \%$ (very high) - is a characteristic feature of intraplate rocks.

We have received fair low isotopic data ${ }^{87} \mathrm{Sr} /{ }^{86} \mathrm{Sr}$ (ISr) in two samples of intraplate rocks of the second type - trachyandesites $\mathrm{R} 70-2-0.7039 \div 0,2$ (high $\mathrm{K} / \mathrm{Rb}=393$ ) and trachybasalt $\mathrm{R} 71-4-0.70489 \div 0,18$ $(\mathrm{K} / \mathrm{Rb}=375$, fig. 4). For subduction-related calc-alkaline andesite of stratovolcano Bazman, sample R-25 was determined a rather low value $\mathrm{ISr}=0.70456 \div 0.05, \mathrm{~K} / \mathrm{Rb}=250$ (tab. 1). Isotopic data of these our intraplate rocks differ from collisional and subduction-related rocks from Anatolia, Turkey (Khain, 2001; Imamverdiyev, 2008 etc.). Igneous rocks of the volcanic rocks are fully differentiated series of the regional known Sahand - Bazman belt. Known mainly andesite stratovolcanoes in this belt are: Bazman with a height 3490m and Taftan - 3940m (old mark was 4042m). Old 0.7049 isotopic date for a 'volcanite' of an unnamed volcano in a desert was reported by Canadian team (Camp, Griffis, 1982).

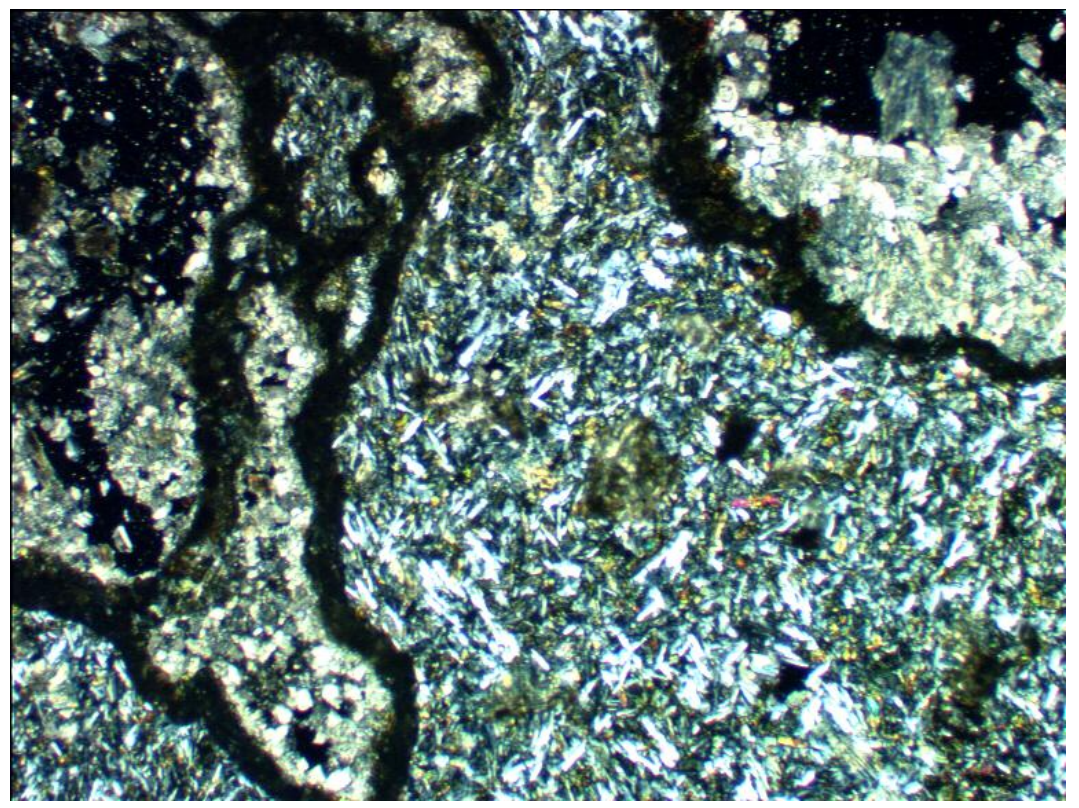

Fig3. Thin section in XPL. R-70 sample. Carbonate-bearing volcanite in Zabol area associated with real carbonatite in Hanne shin structure, Afghanistan. Carbonate is in individual areas of thin section, left and upper right. Up to 33.0 wt.\% of CaO in same $R-70$ group volcanites.

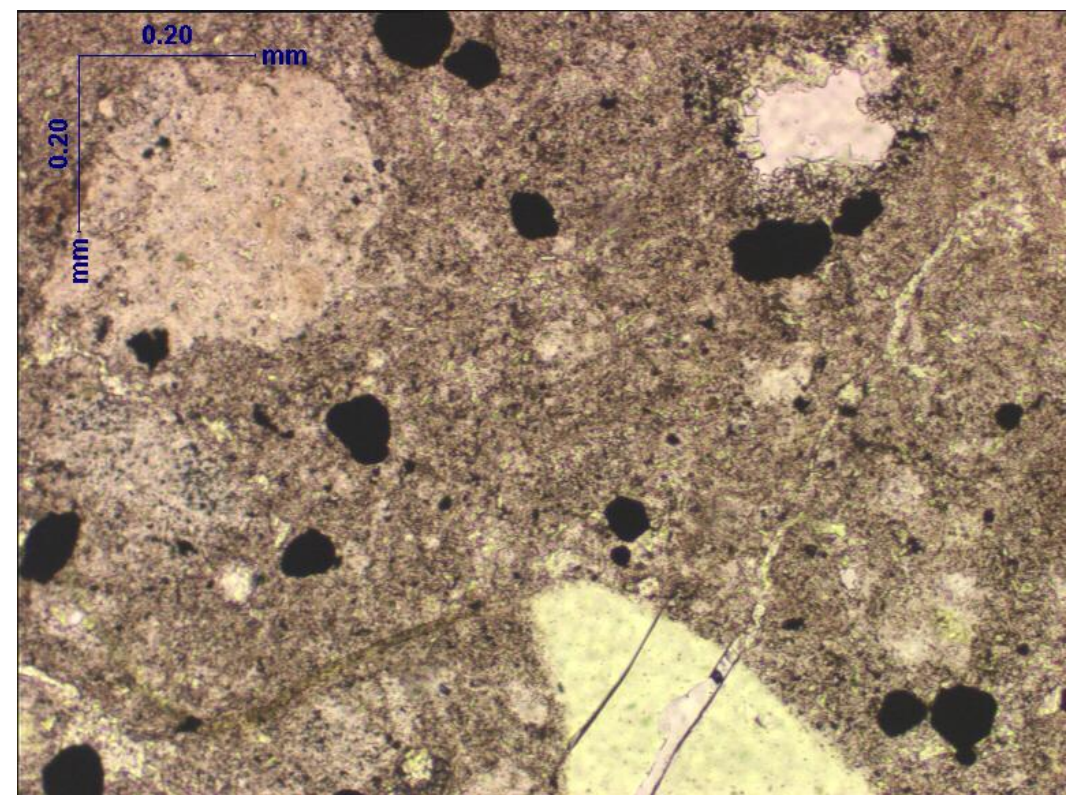

Fig4. Thin section in PPL. R-82 sample, Quaternary? calk-alkaline rhyolite. Bazman stratoVolcano (N-Q age). Quartz - down of thin section. Dark grains - ore minerals. 
Some New and Previous Materials on Alpine Magmatism, Tectonics, Melt and Fluid Inclusions, and Metallogeny in Eastern Iran

Table1. Major- and trace-element composition in the rocks studied

\begin{tabular}{|c|c|c|c|c|c|c|c|c|c|}
\hline Sample & 1 & 2 & 3 & 4 & 5 & 6 & 7 & 8 & 9 \\
\hline $\mathrm{SiO}_{2}$ & 48.17 & 57.80 & 54.50 & 54.00 & 60.69 & 65.39 & 65.10 & 85.00 & 58.67 \\
\hline $\mathrm{TiO}_{2}$ & 2.20 & 1.31 & 1.87 & 1.52 & 0.36 & 0.42 & 0.51 & 0.60 & 1.70 \\
\hline $\mathrm{Al}_{2} \mathrm{O}_{3}$ & 3.80 & 17.48 & 15.94 & - & 15.32 & 13.71 & 15.54 & 4.00 & 15.13 \\
\hline $\mathrm{Fe}_{2} \mathrm{O}_{3}$ & 9.32 & 4.37 & 6.39 & 6.25 & 2.70 & 3.25 & 2.42 & 3.21 & 6.69 \\
\hline $\mathrm{FeO}$ & 2.56 & 1.07 & 0.40 & - & 2.07 & - & 2.32 & 1.10 & 2.19 \\
\hline $\mathrm{MnO}$ & 0.14 & 0.09 & 0.09 & 0.08 & 0.09 & 0.057 & 0.13 & 0.02 & 0.09 \\
\hline $\mathrm{MgO}$ & 5.75 & 2.27 & 3.37 & - & 3.65 & 1.39 & 1.72 & 0.52 & 2.28 \\
\hline $\mathrm{CaO}$ & 8.98 & 7.10 & 7.58 & 7.40 & 3.90 & 2.08 & 2.80 & 0.29 & 1.77 \\
\hline $\mathrm{Na}_{2} \mathrm{O}$ & 4.93 & 5.11 & 5.81 & - & 3.64 & 2.87 & 3.36 & 0.28 & 5.06 \\
\hline $\mathrm{K}_{2} \mathrm{O}$ & 1.31 & 1.42 & 1.73 & 1.09 & 4.38 & 4.51 & 4.59 & 0.21 & 2.05 \\
\hline $\mathrm{P}_{2} \mathrm{O}_{5}$ & 0.23 & 0.61 & 1.05 & - & 0.31 & 0.11 & 0.20 & 0.09 & 0.30 \\
\hline $\mathrm{Rb}$ & 30 & 19 & 20 & 15 & 145 & 117 & 109 & 7 & 47 \\
\hline $\mathrm{Ba}$ & 375 & 293 & - & 292 & 1230 & 577 & 1597 & 390 & 557 \\
\hline $\mathrm{Sr}$ & 1185 & 912 & 4470 & 950 & 870 & 232 & 359 & 440 & 263 \\
\hline $\mathrm{Ni}$ & 86 & 53 & 58 & 59 & 50 & 7 & 13 & 10 & 44 \\
\hline Co & 33 & 14 & - & - & 12 & 5 & 6 & 4 & 21 \\
\hline $\mathrm{Cr}$ & 64 & 60 & 38 & $<64$ & 50 & 16 & 18 & 11 & 72 \\
\hline $\mathrm{V}$ & 220 & 95 & - & - & 81 & 63 & 54 & 55 & 107 \\
\hline $\mathrm{Cu}$ & 63 & 65 & 64 & 77 & 69 & 15 & 11 & 17 & 33 \\
\hline $\mathrm{Zn}$ & 113 & 88 & 113 & 98 & 32 & 40 & 57 & 8 & 82 \\
\hline $\mathrm{Pb}$ & 5 & 20 & 51 & 5 & 20 & 27 & 22 & 20 & 10 \\
\hline $\mathrm{Zr}$ & 283 & 232 & 339 & 217 & 96 & 158 & 246 & 136 & 219 \\
\hline $\mathrm{Y}$ & 25 & 19.5 & 25 & 15 & 15 & 11 & 29 & 13 & 23 \\
\hline $\mathrm{Nb}$ & 23 & 17 & 19 & - & 5.8 & 8 & 12 & 6 & 30 \\
\hline $\mathrm{Sc}$ & 19 & 10.7 & - & 26.2 & 10 & - & - & 6.5 & 10 \\
\hline Th & 3 & 3.65 & - & 4.84 & 12 & - & 16.7 & 1 & 12 \\
\hline $\mathrm{U}$ & 1.2 & 0.99 & - & 1.31 & 1 & - & 4.62 & 3 & 3 \\
\hline $\mathrm{La}$ & 44 & 32.4 & - & 30 & 18 & - & 34.0 & 15 & 35.2 \\
\hline $\mathrm{Ce}$ & 101 & 68.3 & - & 63 & 32 & - & 64.5 & 28 & 64.2 \\
\hline $\mathrm{Nd}$ & - & 31.4 & - & - & - & - & 27 & - & 25.0 \\
\hline $\mathrm{Sm}$ & - & 6.00 & - & - & - & - & 5.6 & - & 5.1 \\
\hline $\mathrm{Eu}$ & - & 2.11 & - & - & - & - & 1.3 & - & 1.9 \\
\hline $\mathrm{Gd}$ & - & 5.08 & - & - & - & - & 4.1 & - & 4.8 \\
\hline $\mathrm{Tb}$ & - & 0.78 & - & - & - & - & - & - & 0.9 \\
\hline $\mathrm{Er}$ & - & 1.64 & - & - & - & - & 1.9 & - & 1.6 \\
\hline $\mathrm{Yb}$ & - & 1.26 & - & - & - & - & 1.7 & - & 1.6 \\
\hline $\mathrm{K} / \mathrm{Rb}$ & 560 & 620 & 586 & 581 & 245 & 307 & 350 & 230 & 350 \\
\hline
\end{tabular}

1 and 2 - trachybasalt (sample R71-4) and trachyandesite (sample R70-2) correspondently, Haji lake, Neogene (?), Afghan block, 3 - trachyandesite, Baluchestan, Iran (Camp, Griffis, 1982), 4 - trachyandesite, R75wp, Lut block, 5 - syenite, Lar intrusiion with Cu-Au mineralization, Miocene(?) 6 - K-dacite, R75, Lut block, and 7 trahydacite, standard, Kurama Ridge Middle Tien Shan, Karamazar, Tajikistan, Late Carboniferous - Early Permian, using data and extrapolation from (Rusinov, Kovalenker, 1991; Razdolina, Moralev et al., 1993; Mamajanov, 2005; Romanko et al., 1989) 8 - leucoRhyolite with many quartz grains and some ore minerals sulfides, R-82, East Bazman volcano, Quarternary(?), 9 - trachyandesite, continental rift, standard, Proterozoic, Pechenga area, Fennoscandian or Baltic shield, by Romanko et al., 1989.

Table2. Chemistry of melt inclusions glass (wt \%) in plagioclase (1, 3), host mineral (2, 4), host acid Kvolcanite (5), rhyolite from Bazman stratovolcano, and plagioclase standards (7-9) due to A. Betekhtin, 1953.

\begin{tabular}{|c|c|c|c|c|c|c|c|c|c|c|c|c|c|}
\hline Sample & $\mathrm{SiO}_{2}$ & $\mathrm{TiO}_{2}$ & $\mathrm{Al}_{2} \mathrm{O}_{3}$ & $\mathrm{FeO}$ & $\mathrm{MnO}$ & $\mathrm{MgO}$ & $\mathrm{CaO}$ & $\mathrm{Na}_{2} \mathrm{O}$ & $\mathrm{K}_{2} \mathrm{O}$ & $\mathrm{P}_{2} \mathrm{O}_{5}$ & $\mathrm{Cl}$ & $\mathrm{S}$ & $\mathrm{Sum}$ \\
\hline 1 & 74.77 & 0.19 & 12.94 & 0.58 & 0.08 & 0.12 & 1.52 & 3.88 & 3.93 & 0.26 & 0.00 & 0.01 & 98.28 \\
\hline 2 & 58.69 & 0.01 & 24.77 & 0.23 & 0.00 & 0.01 & 6.68 & 7.22 & 0.49 & 0.00 & 0.00 & 0.01 & 98.11 \\
\hline 3 & 74.48 & 0.15 & 14.53 & 0.53 & 0.04 & 0.10 & 1.69 & 3.02 & 4.10 & 0.00 & 0.01 & 0.01 & 98.66 \\
\hline 4 & 58.36 & 0.00 & 24.71 & 0.28 & 0.02 & 0.05 & 7.15 & 6.90 & 0.46 & 0.04 & 0.00 & 0.01 & 97.98 \\
\hline 5 & 65.39 & 0.42 & 13.71 & 2.93 & 0.06 & 1.39 & 2.08 & 2.87 & 4.51 & 0.11 & - & - & - \\
6 & 85.00 & 0.60 & 4.50 & 3.98 & 0.02 & 0.52 & 0.29 & 0.28 & 0.21 & 0.09 & - & & - \\
7 & 58.16 & - & 26.57 & - & - & - & 8.35 & 6.92 & - & - & - & & - \\
8 & 56.05 & - & 28.01 & - & - & - & 10.1 & 5.89 & - & - & - & & \\
\hline 9 & 62.43 & - & 23.70 & - & - & - & 5.03 & 8.84 & - & - & - & & \\
\hline
\end{tabular}

1, 3 - melt inclusions glasses in plagioclase, 2, 4 - host minerals, 5 - hosted $\mathrm{Hi}$-K-volcanite, sample R-75, 6 leucorhyolite from stratovolcano Bazman, Quaternary(?), 7-9 plagioclase standards: 7 - andesite, $\mathrm{SiO}_{2}=58.16$, empirical formula - $\mathrm{Na}_{0.6} \mathrm{Ca}_{0.4} \mathrm{Al}_{1.4} \mathrm{Si}_{2.6} \mathrm{O}_{8}$, chemical formula andesite - $(\mathrm{Na}, \mathrm{Ca})(\mathrm{Si}, \mathrm{Al})_{4} \mathrm{O}_{8}$, Webmineral.com, 8 - 9 - plagioclase theoretical composition: $A n_{50}(8)$ and $A n_{25}$ (9), by A. Betekhtin, Moscow, 1953. 
Some New and Previous Materials on Alpine Magmatism, Tectonics, Melt and Fluid Inclusions, and Metallogeny in Eastern Iran

Table3. Sum of gases by thermobarogeochemistry (cub. $\mathrm{cm} / \mathrm{kg}$ )

\begin{tabular}{|c|c|c|}
\hline Sample & $\begin{array}{l}\text { Sum of gase (Cubic } \\
\mathrm{cm} / \mathrm{kg})\end{array}$ & Rock, age, notes \\
\hline 1. R26 & 0.933 & $\begin{array}{c}\text { subvolcanites and shallow intrusions, West Taftan volcano, diorites, probably } \\
\text { Miocene }\end{array}$ \\
\hline 2. R38 & 1.022 & Lar intrusion, Oligocene-Miocene \\
\hline 3. R61 & 0.401 & ophiolites, Cretaceous \\
\hline 4. R85 & 0.655 & ophiolites, Cretaceous \\
\hline 5. R35 & 12.942 & $\begin{array}{l}\text { Subvolcanites intruding CARBONATIC rocks, West Taftan stratovolcano, } \\
\text { maximal contain, probably Oligocene-Miocene. Highest content. }\end{array}$ \\
\hline 6. R66 & 1.262 & $\begin{array}{c}\text { Young } \mathrm{Cu}-\mathrm{Zn}-\mathrm{Pb} \text { mineralization with Au and } \mathrm{Ag} \text {, Taftan stratovolcano, probably } \\
\text { Quaternary }\end{array}$ \\
\hline
\end{tabular}

Sum of gases includes H2, O2, N2, CO2, CH4, C2H6, C3H8, C4H10, C5H12, and C6H14. Temperature of $\mathrm{Au}$ mineralization is $220-278 \mathrm{oC}$, Oligocene-Quaternary, important Lar intrusive massif with Au up to $25.4 \mathrm{ppm}, \mathrm{T}=220-2260 \mathrm{C}$ by analyst R. Mudrogova, VNIIYG GB or Nuclear geophysics Institute, Moscow region (E. Romanko et al., 2000). Maximum of gases are in subvolcanites intruding CARBONATIC rocks. Minimum of gases are in ophiolite mélange rocks.

Table4. ${ }^{87} \mathrm{Sr}{ }^{86} \mathrm{Sr}$ (ISr) isotopic data from the rocks

\begin{tabular}{|c|c|c|}
\hline Sample & ${ }^{87} \mathrm{Sr} /{ }^{86} \mathrm{Sr}$ & Rock, mineral, age, notes \\
\hline 1. & $0,7039+-0,0002$ & $\begin{array}{l}\text { Trachyandesite, sample R-70-2, Hilmand (Afghan) block, maybe } \\
\text { Neogene }\end{array}$ \\
\hline 2. & $0,70489+-0,00018$ & $\begin{array}{c}\text { trachybasalt, R71-4, lake Haji area, Hilmand (Afghan) block, maybe } \\
\text { Neogene }\end{array}$ \\
\hline 3. & $0,70456+-0,00005$ & $\begin{array}{c}\text { calk-alkaline basic andesite, R-25-1, East Bazman volcano, Neogene- } \\
\text { Quaternary }\end{array}$ \\
\hline 4. & 0,7049 & 'volcanite' by Camp and Griffis, 1982, No data about age \\
\hline 5. & $0,7047+-0,0003$ & biotite from trachybasalt, sample 64, Shurab - Galecha, Eocene \\
\hline 6. & $0,7048+-0,0003$ & dacite, sample 166, Eocene \\
\hline 7. & 0,7051 & andesite, sample 206, Eocene \\
\hline 8. & 0,7055 & biotite from andesite, sample 203, Cheh-meh-Huri, Eocene \\
\hline 9. & 0,7059 & andesite, sample 193-A, no age data \\
\hline 10. & 0,7051 & biotite from dacite, sample 143 , Gazu area, no age \\
\hline 11. & 0,7043 & granodiorite, sample 146, no age data \\
\hline 12. & 0,7045 & granodiorite, sample 151 , no age data \\
\hline 13. & $0,7051+-0.0003$ & biotite from granodiorite, Gazu area, Campanian \\
\hline 14. & $0,7048+-0,0003$ & biotite from dacite, Shurab-Galecha, Eocene \\
\hline 15. & $0,7056+-0.0002$ & plagioclase from dacite, Eocene \\
\hline 16. & $0,7065+-0.0003$ & biotite from dacite, Kuh-Berg, Eocene \\
\hline 17. & $0,7070+-0.0003$ & granodiorite, Sor-Kuh, Middle Jurassic \\
\hline 18. & $0,7041+-0.0001$ & Late Cenozoic magma, ENd= +4.1+- 0.2, Great Caucasus \\
\hline 19. & 0,7040 & Late Cenozoic magma, ENd= +3, Great Caucasus \\
\hline
\end{tabular}

1-3 - author's data, 4 - after (Camp, Griffis, 1982), 5-9 - Lut block, immediately west from East Iranian zone, after Sandwall E., Turkell N. Zor E. et al., 2003; 18-19-Geat Caucasus, courtesy of I. Chernyshev, S. Bubnov, A. Lebedev et al., IGEM, RAS, Moscow.

Table5. Composition of rock-forming and accessory minerals

\begin{tabular}{|c|c|c|c|c|c|c|c|c|c|c|c|c|c|}
\hline Sample & 1 & 2 & 3 & 4 & 5 & 6 & 7 & 8 & 9 & 10 & 11 & 12 & 13 \\
\hline $\mathrm{SiO}_{2}$ & 55.45 & 54.11 & 55.20 & 43.79 & 44.65 & 46.25 & 46.87 & 53.86 & 54.54 & 64.49 & 68.94 & 68.61 & 0.018 \\
$\mathrm{TiO}_{2}$ & 0.26 & 0.19 & 0.22 & 1.88 & 1.71 & 1.84 & 1.36 & - & - & 0.26 & - & 0.13 & 29.79 \\
$\mathrm{Al}_{2} \mathrm{O}_{3}$ & 1.51 & 1.52 & 1.50 & 11.37 & 7.88 & 7.69 & 6.40 & 29.51 & 28.22 & 17.82 & 17.20 & 17.85 & 0.02 \\
$\mathrm{Fe}_{2} \mathrm{O}_{3}$ & - & - & - & - & - & - & - & - & - & - & - & - & - \\
$\mathrm{FeO}$ & 11.66 & 15.73 & 12.7 & 13.68 & 13.05 & 13.39 & 15.00 & - & - & 2.79 & 1.12 & 0.96 & 62.11 \\
$\mathrm{MnO}$ & 0.24 & 0.34 & 0.28 & 0.28 & 0.27 & 0.24 & 0.03 & - & - & 0.16 & - & - & 0.17 \\
$\mathrm{MgO}$ & 28.21 & 27.01 & 28.14 & 15.16 & 14.23 & 15.32 & 13.68 & - & - & 0.85 & 0.15 & 0.15 & 1.60 \\
$\mathrm{CaO}$ & 1.89 & 1.16 & 1.93 & 10.55 & 10.48 & 10.42 & 11.51 & 8.97 & 9.99 & 1.87 & 0.47 & 0.70 & 0.08 \\
$\mathrm{Na}_{2} \mathrm{O}$ & 0.32 & - & - & 2.08 & 1.31 & 1.48 & 1.40 & 5.55 & 5.59 & 9.90 & 7.09 & 7.55 & - \\
$\mathrm{K}_{2} \mathrm{O}$ & - & - & - & 0.49 & 0.38 & 0.44 & 0.75 & 0.28 & 0.34 & 1.78 & 4.96 & 3.71 & - \\
$\mathrm{P}_{2} \mathrm{O}_{5}$ & 0.18 & - & - & - & - & - & - & - & - & - & 0.22 & 0.22 & - \\
\hline
\end{tabular}


Some New and Previous Materials on Alpine Magmatism, Tectonics, Melt and Fluid Inclusions, and Metallogeny in Eastern Iran

1-3 - Orthopyroxenes, 2-3 - standard bronzites, orthopyroxenes, 2 - from andesite and 3 - from Hb-norite, by H. Kuno,1964; 4-7-amphiboles; 8-9-plagioclases; 10-12-alkali feldspars; 13 -magnetite.

Table6. Major elements composition in the rocks

\begin{tabular}{|c|c|c|c|c|c|c|c|c|c|c|c|c|c|}
\hline Sample & 1 & 2 & 3 & 4 & 5 & 6 & 7 & 8 & 9 & 10 & 11 & 12 & 13 \\
\hline $\mathrm{SiO}_{2}$ & 48.17 & 49.0 & 52.76 & 54.50 & 56.95 & 57.80 & 35.10 & 44.26 & 46.10 & 56.7 & 60.69 & 61.79 & 85.00 \\
$\mathrm{TiO}_{2}$ & 2.20 & 1.69 & 1.11 & 1.87 & 1.27 & 1.31 & 0.74 & 0.81 & 0.49 & 0.60 & 0.36 & 0.52 & 0.60 \\
$\mathrm{Al}_{2} \mathrm{O}_{3}$ & 13.80 & 14.1 & 17.44 & 15.94 & 16.40 & 17.48 & 13.48 & 12.70 & 10.30 & 11.1 & 15.32 & 17.10 & 4.05 \\
$\mathrm{Fe}_{2} \mathrm{O}_{3}$ & 9.32 & 9.10 & 3.14 & 6.39 & 5.28 & 4.37 & 7.53 & 4.81 & 5.10 & 4.90 & 2.70 & 1.16 & 2.51 \\
$\mathrm{FeO}$ & 2.56 & -0.11 & 5.40 & 0.40 & 0.46 & 1.07 & 0.73 & 0.87 & - & -0.10 & 2.07 & 3.53 & 1.21 \\
$\mathrm{MnO}$ & 0.14 & 9.23 & 0.13 & 0.09 & 0.08 & 0.09 & 0.16 & 0.12 & 0.08 & 4.85 & 0.09 & 0.10 & 0.02 \\
$\mathrm{MgO}$ & 5.73 & 7.72 & 5.55 & 3.37 & 3.35 & 2.27 & 5.46 & 6.60 & 9.00 & 12.0 & 3.65 & 3.04 & 0.37 \\
$\mathrm{CaO}$ & 8.98 & 3.06 & 8.62 & 7.58 & 6.80 & 7.10 & 26.66 & 17.10 & 15.86 & 1.84 & 3.90 & 5.25 & 1.55 \\
$\mathrm{Na}_{2} \mathrm{O}$ & 4.93 & 1.84 & 3.46 & 5.81 & 5.33 & 5.11 & 0.80 & 2.96 & 0.86 & 1.95 & 3.64 & 4.11 & 0.28 \\
$\mathrm{~K}_{2} \mathrm{O}$ & 1.31 & 0.40 & 1.31 & 1.73 & 1.50 & 1.42 & 0.10 & 0.42 & 2.36 & 0.12 & 4.38 & 1.58 & 0.21 \\
$\mathrm{P}_{2} \mathrm{O}_{5}$ & 1.11 & & 0.40 & 0.51 & 0.59 & 1.05 & 0.16 & 0.38 & 0.12 & & 0.31 & 0.19 & 0.03 \\
\hline
\end{tabular}

1-10 - Hilmand (Afghan) block: 1-3 - trachybasalts, 11 - syenite, Lar massif, 12 - 13 - Bazman volcano, Neogene-Quaternary, author's data; 2, 7,10 - data by A. Houshmandzadeh and M.A.A. Nogol Sadat et al., 3 and 4-(Camp, Griffis, 1982), '-' not determined.

Table7. Rare Earth Elements (REE) in the rocks studied and standards

\begin{tabular}{|c|c|c|c|c|c|c|c|c|c|c|}
\hline Sample & 1 & 2 & 3 & 4 & 5 & 6 & 7 & 8 & 9 & 10 \\
\hline $\mathrm{La}$ & 32.4 & 32.1 & 44.8 & 18.6 & 35.2 & 34 & 63 & 78 & 31.3 & 23 \\
\hline $\mathrm{Ce}$ & 68.3 & 69.3 & 91.9 & 37.7 & 64.2 & 71 & 115 & 50 & 50.8 & 43 \\
\hline $\mathrm{Pr}$ & 8.23 & 8.05 & 9.80 & 4.32 & - & - & - & - & - & - \\
\hline $\mathrm{Nd}$ & 31.4 & 32.9 & 37.8 & 17.7 & 25.0 & 43 & 70 & 63 & 21.3 & \\
\hline $\mathrm{Sm}$ & 6.00 & 5.98 & 7.24 & 3.92 & 5.1 & 10 & 17 & 12 & 4.09 & 4.72 \\
\hline $\mathrm{Eu}$ & 2.11 & 1.83 & 1.31 & 1.23 & 1.9 & 3.0 & 4.5 & 4.0 & 1.26 & 1.56 \\
\hline $\mathrm{Gd}$ & 5.08 & 5.55 & 6.19 & 4.20 & 4.8 & 7.5 & 11 & 10 & 3.42 & \\
\hline $\mathrm{Tb}$ & 0.78 & 0.71 & 0.70 & 0.54 & - & - & - & - & 0.55 & 1.93 \\
\hline $\mathrm{Dy}$ & 3.20 & 3.13 & 3.76 & 3.50 & - & - & - & - & - & \\
\hline $\mathrm{Ho}$ & 0.68 & 0.57 & 0.64 & 0.69 & - & - & - & - & - & \\
\hline $\mathrm{Er}$ & 1.26 & 1.40 & 1.93 & 2.21 & 1.6 & 2.8 & 3.7 & 2.9 & 1.79 & \\
\hline $\mathrm{Tm}$ & 0.31 & 0.26 & 0.26 & 0.32 & - & - & - & - & - & \\
\hline $\mathrm{Yb}$ & 1.26 & 1.10 & 1.74 & 2.23 & 1.6 & 1.8 & 2.4 & 2.8 & 1.94 & 1.97 \\
\hline $\mathrm{Lu}$ & 0.34 & 0.23 & 0.25 & 0.34 & - & - & - & - & - & \\
\hline
\end{tabular}

1-4 - intraplate rocks in West Baluchestan: 1-2 - trachyandesites, Neogene (r70-2 and r70-23 samples, analytics by A. Housmandzadeh and M.A.A. Nogol Sadat support); Helmand basin, 3-4 - subalkaline rocks, Lut block (r75-1 and r75-2); 1-4 - analytics by A. Housmandzadeh and M.A.A. Nogol Sadat support; 5-trachyandesite, standard, continental rift, Paleoproterozoic, Kuetsjarvi unit, Pechenga zone, Fennoscandian Shield by A. Romanko et al.; 6-8 - basalt and dolerite (intraplate standard rocks), continental rift, Jurassic, Karoo formation, Save-Limpopo rift, Zimbabwe, E. and A. Romanko; 9 - trachyandesite, Eocene, subduction-related setting, sample BH-13 from a well, Talmessi deposit, Central Iran, courtesy of H. Bagheri, 10- trachybasalt, sill, sample Ta-39, Eocene, Lesser Caucasus, Imamverdiyev, 2010.

Table8. Composition of glass in acid volcanite R-82, East Bazman stratovolcano, $T$ crystallization $=6900$, content of $\mathrm{H} 2 \mathrm{O}$ - more than $6 \mathrm{wt} \%$ by different methods.

\begin{tabular}{|c|c|c|c|c|c|c|c|c|c|c|c|c|c|c|}
\hline Sample & $\mathrm{SiO}_{2}$ & $\mathrm{TiO}_{2}$ & $\mathrm{Al}_{2} \mathrm{O}_{3}$ & $\mathrm{FeO}$ & $\mathrm{MnO}$ & $\mathrm{MgO}$ & $\mathrm{CaO}$ & $\mathrm{Na}_{2} \mathrm{O}$ & $\mathrm{K}_{2} \mathrm{O}$ & $\mathrm{P}_{2} \mathrm{O}_{5}$ & $\mathrm{Cl}$ & $\mathrm{S}$ & Sum & $\mathrm{Rastr}$ \\
\hline 1 & 72.70 & 0.10 & 10.88 & 0.68 & 0.05 & 0.08 & 0.68 & 2.49 & 3.69 & 0,04 & 0.11 & 0.02 & 91.52 & $12 \times 12$ \\
\hline 2 & 72.78 & 0.14 & 11.39 & 0.75 & 0.07 & 0.12 & 0.74 & 2.63 & 3.69 & 0,02 & 0.13 & 0.04 & 92.50 & $12 \times 12$ \\
\hline 3 & 72.59 & 0.14 & 11.40 & 0.71 & 0.05 & 0.12 & 0.72 & 2.22 & 3.74 & 0.03 & 0.12 & 0.03 & 91.87 & $20 \times 20$ \\
\hline 5 & 71.44 & 0.07 & 11.10 & 0.71 & 0.06 & 0.13 & 0.77 & 2.64 & 3.57 & 0.04 & 0.16 & 0.01 & 90.70 & $12 \times 12$ \\
\hline 6 & 71.96 & 0.09 & 11.17 & 0.62 & 0.00 & 0.16 & 0.74 & 2.78 & 3.70 & 0.14 & 0.13 & 0.03 & 91.52 & $12 \times 12$ \\
\hline 7 & 72.03 & 0.13 & 11.12 & 0.72 & 0.07 & 0.13 & 0.79 & 2.88 & 3.71 & 0.15 & 0.15 & 0.01 & 91.89 & $12 \times 12$ \\
\hline 8 & 72.61 & 0.06 & 11.31 & 0.72 & 0.00 & 0.13 & 0.71 & 2.83 & 3.75 & 0.12 & 0.16 & 0.02 & 92.42 & $12 \times 12$ \\
\hline
\end{tabular}

1-8 - composition of glass inclusion in Quartz of acid volcanite, rhyolite R-82, East Bazman stratovolcano, T of crystallization $=690 \mathrm{oC}$, High content of $\mathrm{H} 2 \mathrm{O}=6 \mathrm{wt} \%$. There are many sulfides in a sample correlated with higher content of $\mathrm{Cu}, \mathrm{Zn}$ etc. in a sample R-82. Analyses led by V. Prokofiev. 
Some New and Previous Materials on Alpine Magmatism, Tectonics, Melt and Fluid Inclusions, and Metallogeny in Eastern Iran

Table9. Rare, trace (ppm) and major (\%) elements composition

\begin{tabular}{|c|c|c|c|c|c|c|c|c|c|c|c|c|c|c|c|c|c|c|}
\hline sample & $\mathrm{Ni}$ & $\mathrm{Cu}$ & $\mathrm{Zn}$ & Ga & $\mathrm{Pb}$ & $\mathbf{R b}$ & $\mathrm{Sr}$ & $\mathbf{Y}$ & $\mathrm{Zr}$ & $\mathrm{Fe}_{2} \mathrm{O}_{3} \mathrm{t}$ & $\mathbf{K}_{2} \mathbf{0}$ & $\mathrm{CaO}$ & $\mathrm{Ti}_{2}$ & $\mathrm{Cr}_{2} \mathrm{O}_{3}$ & $\mathrm{MnO}$ & $\mathrm{Ba}$ & La & $\mathrm{Ce}$ \\
\hline 1. $75-\mathrm{WP}$ & 59 & 77 & 98 & 14 & 51 & 15 & 950 & 15 & 217 & 6.25 & 1.09 & 7.40 & 1.52 & $<64$ & 0.08 & 292 & 30 & 63 \\
\hline 2. $71-4$ & 77 & 75 & 113 & 13 & 61 & 181 & 1138 & 24 & 245 & 9.96 & 1.10 & 9.73 & 2.49 & 64 & 0.13 & 376 & 40 & 111 \\
\hline 3. $71-42$ & 87 & 631 & 197 & 14 & 51 & 141 & 1097 & 22 & 223 & 9.56 & 1.23 & 10.19 & 2.02 & $<64$ & 0.12 & 375 & 44 & 101 \\
\hline 4. $71-43$ & 82 & 68 & 110 & 14 & 61 & 161 & 1115 & 23 & 234 & 9.8 & 1.16 & 9.73 & 2.49 & 64 & 0.13 & 376 & 40 & 106 \\
\hline 5. $70-32$ & 40 & 131 & 171 & 9 & 26 & 5 & 181 & 18 & 91 & 7.39 & 0.02 & 34.89 & 0.48 & $<64$ & 0.12 & 40 & 13 & 14 \\
\hline 6. $70-4$ & 43 & 166 & 6164 & 46 & 20 & 5 & 505 & 17 & 146 & 4.72 & 0.32 & 21.32 & 0.63 & $<64$ & 0.09 & 155 & 20 & 37 \\
\hline 7. $70-5$ & 162 & 86 & 689 & 10 & 13 & 20 & 751 & 13 & 186 & 5.91 & 1.31 & 10.88 & 1.12 & 0.04 & 0.08 & 310 & 28 & 57 \\
\hline 8. $70-6$ & 136 & 65 & 579 & 11 & 10 & 22 & 782 & 18 & 180 & 5.86 & 130 & 10.47 & 1.14 & 0.03 & 0.07 & 304 & 23 & 55 \\
\hline 9. $70-7$ & 49 & 77 & 786 & 14 & 13 & 16 & 5992 & 15 & 208 & 6.01 & 1.09 & 7.90 & 1.50 & 64 & 0.07 & 319 & 21 & 69 \\
\hline 10. $70-8$ & 42 & 77 & 787 & 13 & 5 & 13 & 31106 & 16 & 205 & 6.24 & 1.11 & 8.04 & 1.52 & $<64$ & 0.07 & 334 & 35 & 64 \\
\hline 11. $70-9$ & 38 & 60 & 83 & 14 & 6 & 14 & 875 & 14 & 183 & 5.11 & 1.55 & 6.54 & 1.27 & $<64$ & 0.07 & 270 & 30 & 69 \\
\hline 12. $70-10$ & 67 & 80 & 93 & 16 & 12 & 16 & 683 & 9 & 100 & 5.62 & 1.46 & 7.87 & 1.53 & $<64$ & 0.08 & 318 & 31 & 68 \\
\hline 13. $70-11$ & 52 & 62 & 292 & 17 & 8 & 16 & 943 & 15 & 215 & 6.21 & 1.30 & 7.04 & 1.64 & $<64$ & 0.08 & 273 & 30 & 58 \\
\hline 14. $70-12$ & 50 & 85 & 589 & 15 & 9 & 17 & 900 & 15 & 205 & 6.10 & 1.47 & 7.63 & 1.38 & $<64$ & 0.08 & 324 & 32 & 68 \\
\hline 15. $70-13$ & 57 & 57 & $7 \quad 79$ & 12 & 14 & 20 & 917 & 17 & 201 & 5.96 & 1.37 & 8.19 & 1.36 & $<64$ & 0.08 & 379 & 35 & 67 \\
\hline 16. $70-14$ & 51 & 60 & 83 & 19 & 8 & 15 & 863 & 18 & 203 & 5.06 & 1.47 & 6.93 & 1.31 & $<64$ & 0.06 & 292 & 28 & 64 \\
\hline 17. $70-15$ & 67 & 80 & 93 & 16 & 12 & 16 & 683 & 9 & 199 & 5.62 & 1.46 & 7.87 & 1.53 & $<64$ & 0.08 & 318 & 31 & 68 \\
\hline 18. $82-5$ & 20 & 70 & D 170 & $0 \quad 18$ & $3-$ & 93 & 52 & 36 & 516 & 7.81 & 4.35 & 0.99 & 1.12 & $<64$ & 0.12 & 781 & 56 & 104 \\
\hline
\end{tabular}

1- 17 - intraplate rocks, Baluchestan and Sistan Province: 1- Lut block (R-75wp, sample of E. Romanko), 2-4 temporary Haji lake, north from Zabol, 5-17 - unnamed volcano in desert, 18 - important calc-alkaline rhyolite $R-82, T$ crystallization $=690 \mathrm{oC}, \mathrm{H} 2 \mathrm{O}=6 \mathrm{wt} \%$, east Bazman stratovolcano, Quaternary ?. "- "means below resolution concentration. XRF is by TEFA-3 techniques.

Table10. ICP-MS data (ppm) on volcanites and ore sample

\begin{tabular}{|c|c|c|c|c|}
\hline 2 & 3 & 4 & 5 & 6 \\
\hline $1 \mathrm{~s} 59$ & $9 \mathrm{~s} 66$ & 3as1 & 9s15 & Mr3S95 \\
\hline 50 & 40 & 45 & 43 & 24 \\
\hline 2,3 & 2,1 & 1,6 & 2,8 & 2,21 \\
\hline 11,3 & 9,7 & 12,1 & 9,7 & 10,8 \\
\hline 4097 & 3786 & 4060 & 3769 & - \\
\hline 137 & 138 & 165 & 138 & 166 \\
\hline 12 & 6,9 & 17 & 11 & 44 \\
\hline 731 & 1025 & 786 & 671 & - \\
\hline 14 & 13 & 18 & 12 & 15,3 \\
\hline 3,6 & 1,8 & 6,5 & 2,4 & 9,25 \\
\hline 38 & 284 & 13709 & 62 & 685 \\
\hline 95 & 125 & 91 & 44 & - \\
\hline 28 & 27 & 27 & 26 & 18 \\
\hline 101 & 69 & 104 & 78 & 131 \\
\hline 915 & 1309 & 814 & 938 & 1077 \\
\hline 18 & 18 & 17 & 17 & 21 \\
\hline 144 & 134 & 130 & 132 & 133 \\
\hline 7,5 & 6,9 & 7,5 & 6,9 & 8,2 \\
\hline 1,1 & 0,74 & 3,1 & 1,0 & \\
\hline 2,4 & 10 & 4,1 & 36 & \\
\hline 639 & 517 & 534 & 512 & \\
\hline 29 & 29 & 26 & 30 & 30 \\
\hline 59 & 58 & 54 & 57 & 59 \\
\hline 7,3 & 7,2 & 6,7 & 7,0 & - \\
\hline 29 & 28 & 27 & 27 & 29 \\
\hline 5,4 & 5,3 & 5,1 & 5,2 & 5,3 \\
\hline 1,6 & 1,6 & 1,6 & 1,6 & 1,47 \\
\hline 5,3 & 5,0 & 5,0 & 5,0 & 4,7 \\
\hline
\end{tabular}


Some New and Previous Materials on Alpine Magmatism, Tectonics, Melt and Fluid Inclusions, and Metallogeny in Eastern Iran

\begin{tabular}{|c|c|c|c|c|}
\hline 0,71 & & & & 0,67 \\
\hline 3,9 & 0,67 & 0,67 & 3,6 & 0,69 \\
\hline 0,75 & 3,7 & 3,6 & 0,71 & 0,88 \\
\hline 2,2 & 0,74 & 0,72 & 2,1 & 2,21 \\
\hline 0,32 & 2,2 & 2,1 & 0,31 & 0,32 \\
\hline 2,2 & 0,31 & 0,30 & 2,1 & 2,10 \\
\hline 0,33 & 2,2 & 2,0 & 0,30 & 0,34 \\
\hline 3,6 & 0,32 & 0,30 & 3,3 & 3,35 \\
\hline 0,47 & 3,4 & 3,2 & 0,43 & 0,83 \\
\hline 1,8 & 0,42 & 0,45 & 0,73 & - \\
\hline 16 & 0,49 & 0,82 & 15 & 10,0 \\
\hline 0,003 & 14 & 14 & 0,085 & - \\
\hline 7,6 & 0,022 & 0,091 & 7,2 & 7,9 \\
\hline 2,4 & 7,3 & 6,9 & 2,0 & 2,03 \\
\hline
\end{tabular}

1-6 - volcanites including Cu-rich ones, Eocene (Pg2)?, Abbas Abad

Cu mining area, NE. Iran, samples of M. Heidari.

Table11. Trace elements in rocks (ppm)

\begin{tabular}{|c|c|c|c|c|c|c|}
\hline Sample & $\mathbf{R b}$ & $\mathbf{S r}$ & Y Zr Nb Ba & 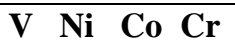 & Sc & As \\
\hline $1 . R 71-4$ & 16 & 1290 & 2527038380 & $24072 \quad 35100$ & 25 & $13<1<1$ \\
\hline 2.R70-8 & 16 & 970 & 1422015380 & $95 \quad 32 \quad 14 \quad 53$ & 14 & $8.9<1 \quad 1.8$ \\
\hline 3.R70-28 & 17 & 970 & $1622016 \quad 340$ & $10036 \quad 1354$ & 13 & $10<1$ \\
\hline 4.R70-27 & 3.8 & 510 & $1213011 \quad 140$ & $8926 \quad 1272$ & 16 & $19<1$ \\
\hline 5.70 .272 & 4.1 & 170 & 16755.5120 & $130202.8 \quad 21$ & 27 & $\begin{array}{lll}17 & 4.2 & 3.6\end{array}$ \\
\hline 6.R28-59 & 145 & 1230 & 131105.8870 & $8150 \quad 1255$ & 15 & $\begin{array}{lll}7.9 & 6.3 & 12\end{array}$ \\
\hline $7.34-24$ & 6.7 & 170 & $23 \quad 858.476$ & $\begin{array}{llll}34 & 7 & 5.3 & 39 \\
\end{array}$ & 5 & $\begin{array}{lll}4.8 & 1 & 14 \\
\end{array}$ \\
\hline
\end{tabular}

R-71-4 - trachybasalt, samples R-70 - trachyandesites and associated intraplate rocks, 28-59- syenite, Lar, intrusive massif, N1?, 34-24 - acid subduction-related dacite, N. Pamirs, Late Permian (P2), V. Novikov, A. Romanko et al, 1992, for comparing. XRF, ppm, Geological Institute, RAS.
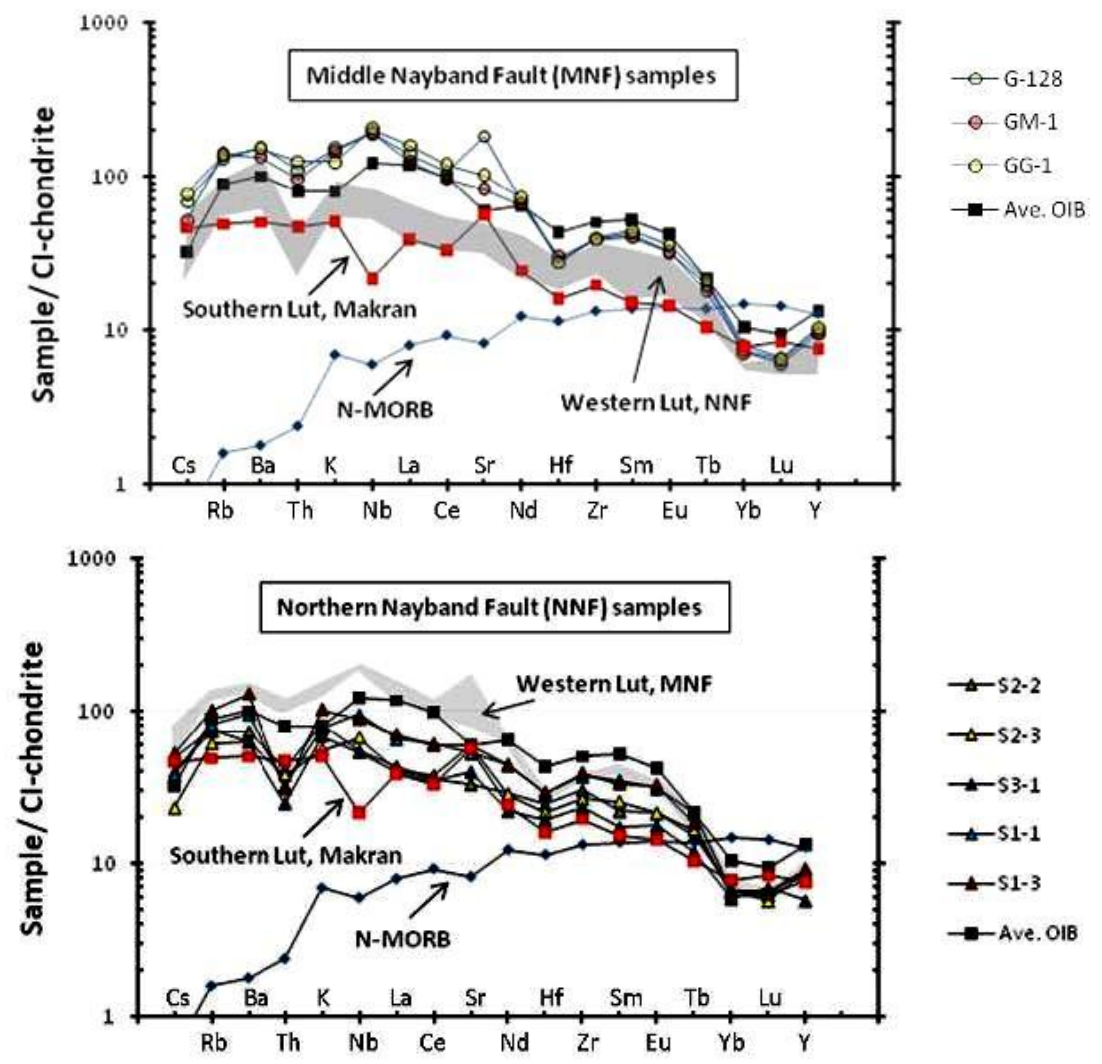

Fig5. The distribution of the contents of rare and trace elements normalized to chondrite composition (Sun, McDonaugh, 1989), using (Saadat S, Stern C.R., 2011). 

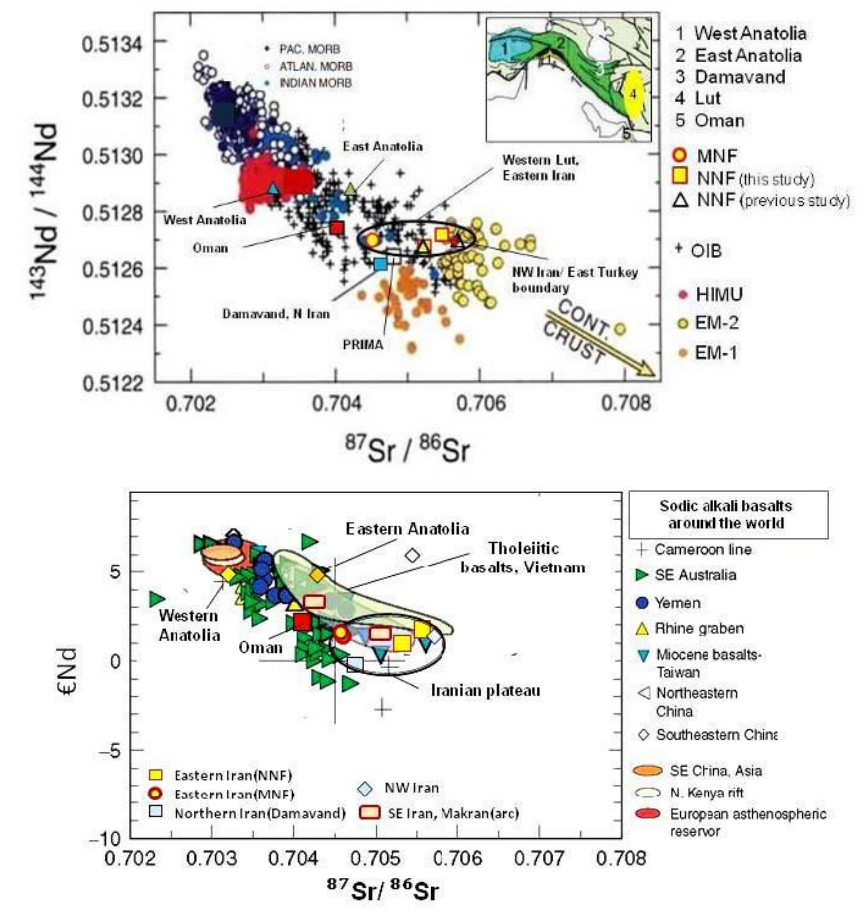

Fig6. Isotope systematics of igneous rocks in the region and standards using (Saadat S, Stern C.R., 2011).

\section{INCLUSIONS}

Melt inclusions in this region were firstly investigated under the leadership of Prof. Prokofiev, IGEM RAS as well as fluid inclusions by E. Romanko et al. in 2000. Some notes and conclusions here are as folllows:

- Melt inclusions are not typical for the African super-plume-related intraplate igneous rocks. Intraplate rocks are confirmed by a tomography of known Ritsema's team (Bull et al., 2009 etc). Also, melt inclusions are not typical or rare for shoshonite series rocks of Abbas Abad area. $\mathrm{T}$ crystallization of melt inclusions in similar Eocene shoshonite series rocks with Fe-skarn mineralization, West Iran is fairly high - ca $300 \mathrm{oC}$ by V. Prokofiev et al.

- unusual fairly high temperature, $1150-1180^{\circ} \mathrm{C}$ - up to $1220^{\circ} \mathrm{C}$ melt inclusions were revealed in plagioclase of subduction-related K-dacite, sample 75-1 by V. Prokofiev et al, 2011 (Prokofiev, 2000; Romanko et al., 2012, Fig. 5 and 6, Tables 2, 8.). This fairly deep, non-calc-alkaline rock was also affected by indirect (?) influence of a huge African super-plume, as proposed. Homogenization occurs under High $\mathrm{T}=1150-1220^{\circ} \mathrm{C}$ (for comparing, for example, $\mathrm{T}$ much lower for acid volcanite of Quaternary Pektusan volcano, Korea, paper of O. Andreeva et al., IGEM RAS, Moscow, 2013). A higher viscosity of a glass provides more inclusions coexistence in a sample.

Maximal concentration on fluid $\mathrm{CH}_{4}$ and other $\mathrm{CH}$-based fluid inclusions were revealed in shallow intrusions on the contact with carbonate-rich host rocks in west Taftan zone; also in important Lar syenite massif with $\mathrm{Cu}-\mathrm{Au}$ mineralization (Table 3, E. Romanko et al., 2000). Opposite, minimal data are in Cretaceous ophiolitic mainly melange rocks.

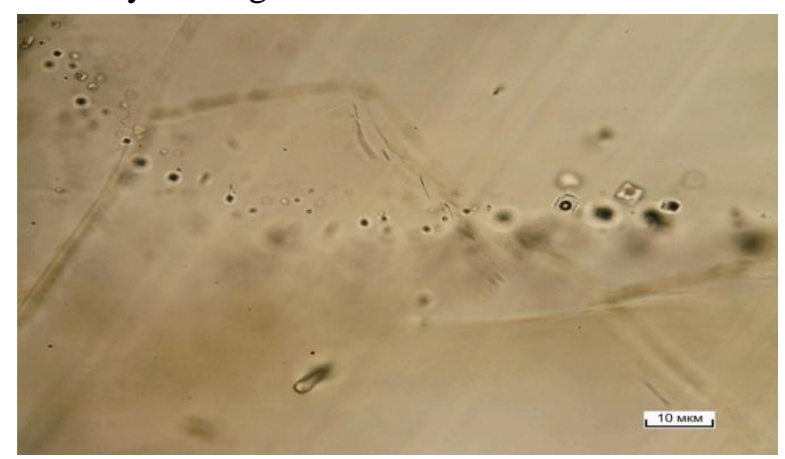

Fig7. Sample N-40. L. Caucasus, for comparison. T of crystallization $=1080-1140^{\circ} \mathrm{C}$. Melt inclusions in Plagioclase (Plg) from trachyandesite. Similar melt inclusions are in similar Alpine volcanites in eastern Iran too. 
Some New and Previous Materials on Alpine Magmatism, Tectonics, Melt and Fluid Inclusions, and Metallogeny in Eastern Iran

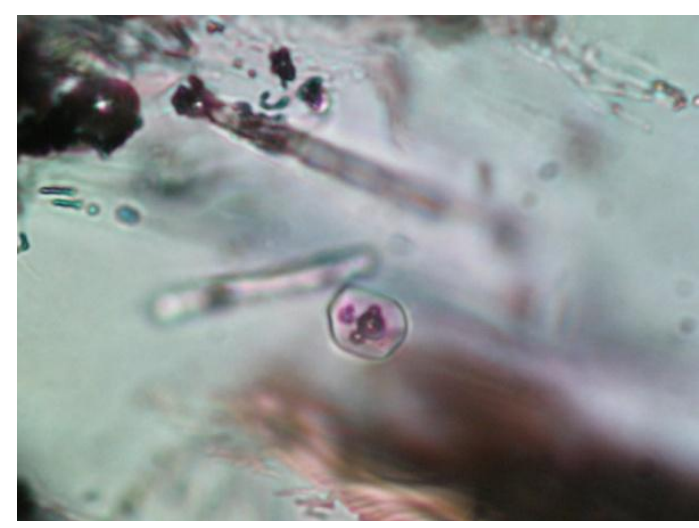

Fig8. Sample R-75ia. Baluchestan and Sistan Province, Iran. T=1150 C. View of melt inclusions in acid glass from Plagioclase

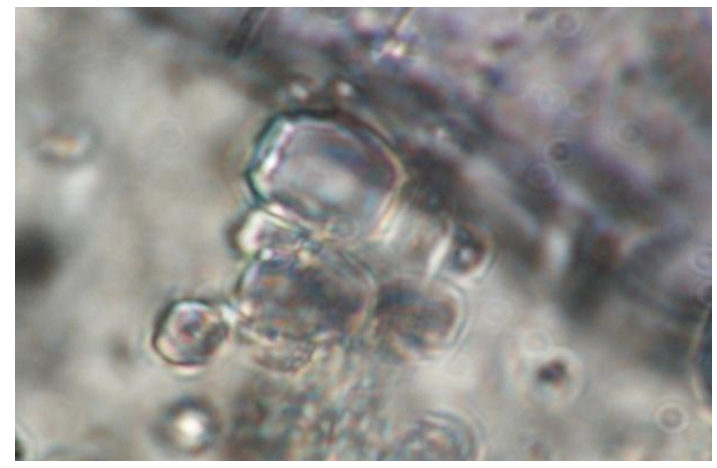

Fig9. Sample R-75ia. T of crystallization is fairly high - 1150-1220oC. Homogenization of melt inclusions.

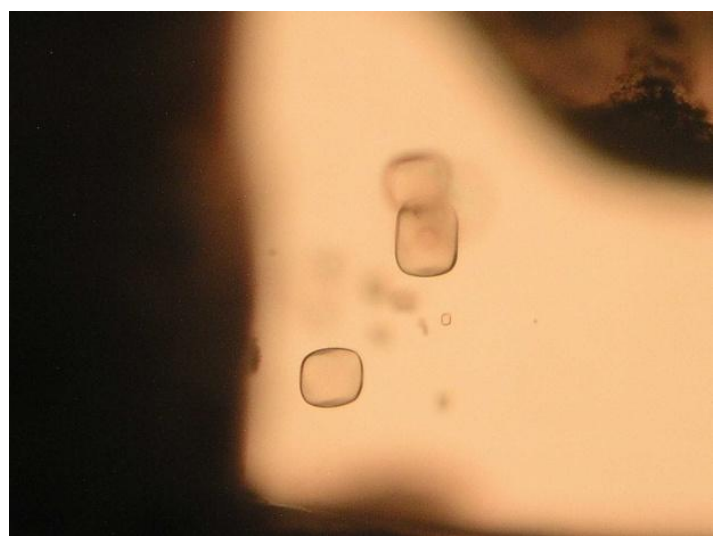

Fig10. Sample R-82. Melt inclusions in Quartz, acid rhyolite, Bazman strato-volcano, T=690oC. H2O content is high $=6 \mathrm{wt} \%$. Naturally chilled melt inclusion. Maximal size of inclusion is ca $60 \mu \mathrm{m}$ or microns.

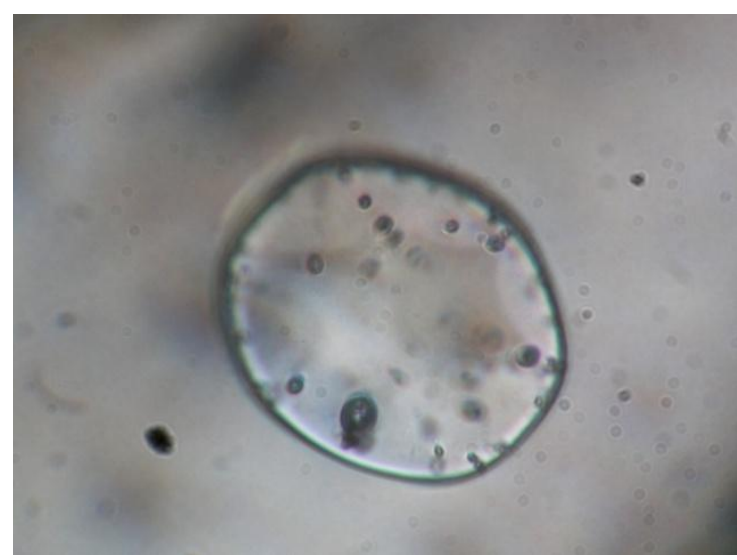

Fig11. Sample R-82. Similar melt inclusion with gas bubbles in Quartz, rhyolite, East Bazman volcano, $T=$ $630 o C$. After next $T=690 \circ C$ gas bubbles will disappear. H2O content is up to 6 wt\%. 
Some New and Previous Materials on Alpine Magmatism, Tectonics, Melt and Fluid Inclusions, and Metallogeny in Eastern Iran

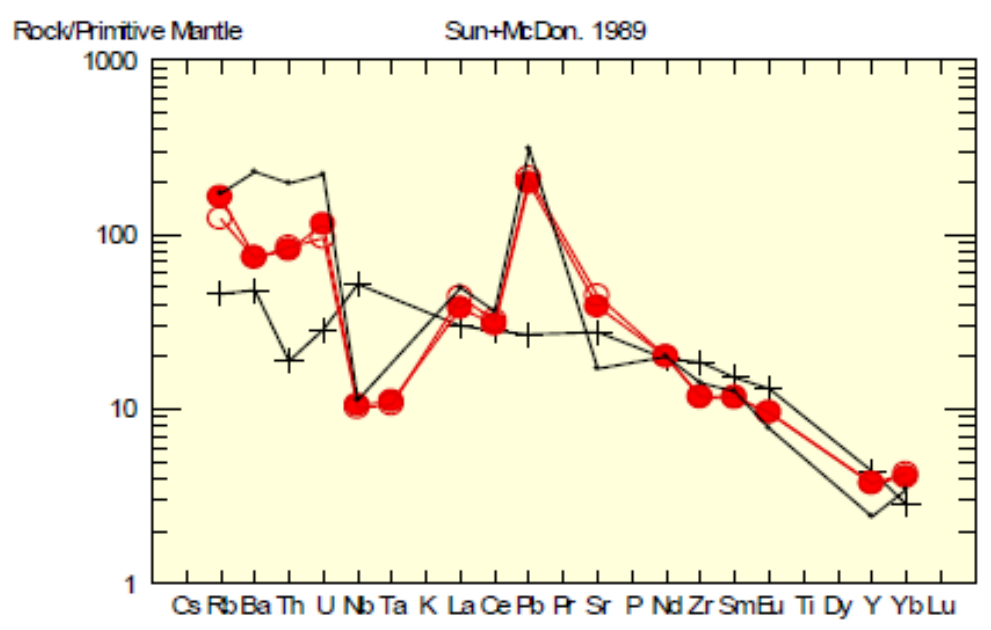

Fig.12. Spider-diagram for non-intraplate rocks, principally other, probably - subduction-related rocks. Circles - shoshonite series rocks, Abbas Abad copper deposit, Central Iran, M. Heidari's samples, cross - intraplate rocks pf Lut block by Saadat et al, 2002, dots - Kurama Mt, Tien Shan, C3-P1 age, analogues of studied shoshonitic - latitic rocks. Usual positive anomaly by $\mathrm{Pb}$. Ta - Nb deficit here is in subduction-related? rocks. For a comparison, example: Cross - principally OTHER intraplate rocks of Lut block by Saadat et al, 2002.

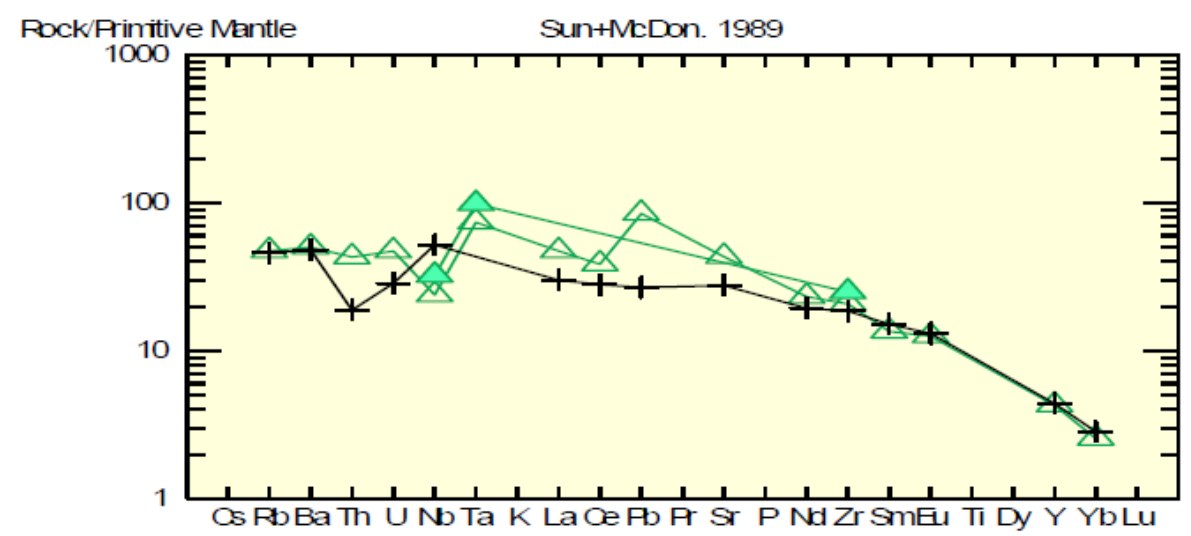

Fig13. Spider-diagram for intraplate rocks of East Iran, typical flat profiles. Sometimes -usual Pb positive anomaly. Triangiulars - R-70 - unnamed volcano (full plot with usual Pb - positive anomaly), $R-71-H a j i$ temporary lake in a desert, not-full profile, no Middle - HREE data here, cross - intraplate rocks of Lut block by Saadat et al, 2002.

Sun+McDon. 1989-REES RockChondrites

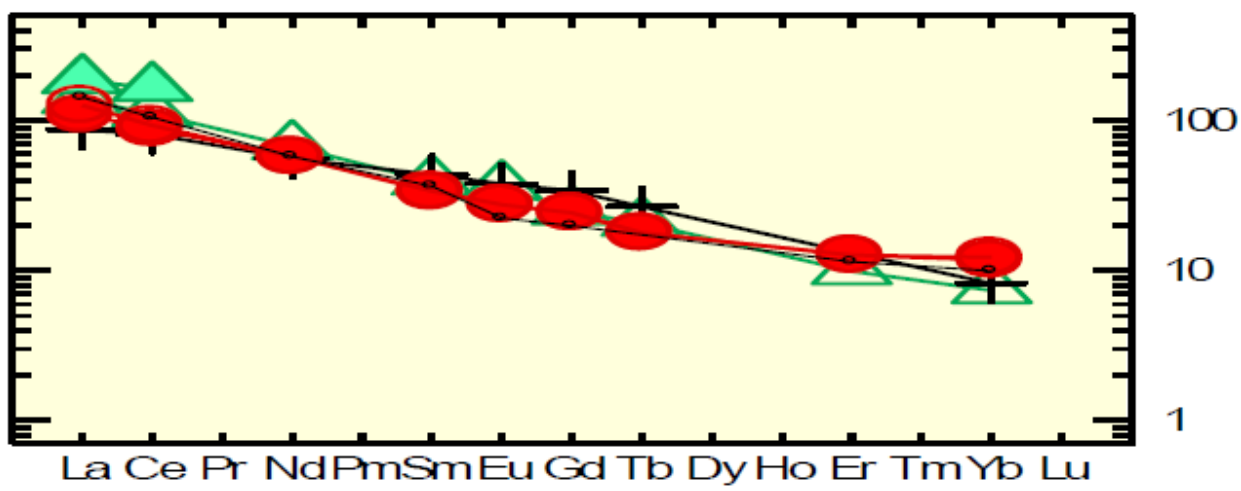

Fig14. Chondrite-normalized REE diagram for Cenozoic intraplate rocks and Eocene (Pg2) subduction-related ones of Iran, $N=6$. Intraplate rocks are: $R-70$ (triangulars), $R-71$ (filled green triangulars), and crosses sample from Lut block (Saadat et al., 2002). Circles - shoshonite - latite series rocks, Eocene (Pg2), Abbas Abad Cu mining area, $N=2$, samples of $M$. Heidari. Dots - rock of shoshonite-latite series, analogue, Kurama Mts, Middle Tien Shan, Late Carboniferous - Early Permian (C3-P1). Absent of Eu-deficit is typical for both intraplate and subduction-related rocks. 
Some New and Previous Materials on Alpine Magmatism, Tectonics, Melt and Fluid Inclusions, and Metallogeny in Eastern Iran

Intraplate rocks were derived from deeper mantle source versus subduction-related Eocene and Late Cenozoic rocks. This is supported by the following:

- Geological and petrographic and mineralogical data

- The general style of petrology and geochemistry of these rocks, we can the same on other regions

- Obvious geochemical materials, for example, the stable high $\mathrm{K} / \mathrm{Rb}=560-586-620$ etc.

The region is expected to at least partial compensation of Pg2-Q aged compression and subductionrelated Magmatism by intraplate magmatism. The latter, according to the imaging may be associated with the tail of the most powerful African superplume (Bull et al., 2009). There is also discussion in modeling - the partial screening of the plume push up plate, which is not an obstacle - it is known that plate moves may not stop movement of the tail superplume in lateral direction.

\section{MetallogeniC NOTES}

Neogene rocks of the Lesser Caucasus is interesting for economic and non-economic metallogeny as:

-New Low-temperature $\mathrm{Au}-\mathrm{Ag}, \mathrm{Hg}$, $\mathrm{As}, \mathrm{Sb}, \mathrm{Cu}-\mathrm{Mo}$ with $\mathrm{Au}, \mathrm{Cu}-\mathrm{Pb}-\mathrm{Zn}$ and $\mathrm{Pb}-\mathrm{Zn}$ deposits and occurrences is proposed here,

-Non-ore raw - tuffs, slags, pumice etc. are of interest too.

Metallogeny of Cenozoic rock of East Iran was studied under the leadership of outstanding regional trio - Dr. Eugene Romanko, Dr. A. Houshmandzadeh, and Dr. M.A.A. Nogole-Sadat.

Calc-alkaline intrusive, extrusive, pyroclastic and volcanogenic-sedimentary rocks here are characterized by a common copper-gold $(\mathrm{Cu}-\mathrm{Au})$ metallogenic profile for Baluchestan and Sistan Province in East Iran, as in the whole Sahand - Bazman volcanic-plutonic belt of Iran. The overwhelming majority of occurrences the study area is associated with magmatic complexes. Such metallogenic types were revealed here as:

- Multi-sulfide (Au-Mo-Cu-Pb-Zn) subvolcanic porphyry type;

- Au-As-Hg-W-Mo-volcanic exhalation one;

-Low-sulfide gold-silver plutonic one;

-Gold-copper (Au-Cu) skarn and plutonic-hydrothermal one (E. Romanko et al., 2000; data by Pars Kani Co, 2003 by Daliran et al., 2005) using also known data on mineralization of different region including former USSR / CIS (Prokofiev et al., 2000; Vikentiev et al., 2004 etc.);

-Sulfide, sulfur, alunite exhalation, surface one;

-Native-copper-sulphide volcanogenic one with zeolites;

-Silver volcanogenic sulphide (+ gold?) one.

Thus, intraplate rocks are strongly specialized in REE, $\mathrm{P}$ (usual process), then in $\mathrm{Sr}, \mathrm{Ba}, \mathrm{U}$, Th due to nowdays materilas. So, tectonic-magmatic, and as revealed E.Romanko - metallogenic zonation in the region was revealed in the region studied (at least in the Central - East Iran). Younger magmatic products are in the northeast of region due to lithosphere subduction and decreasing of Afrocan superplume activity in the same direction. Subduction-related (1 group of rocks) dominated calcalkaline rocks and shoshonites-latites. , and, intraplate African superplume-related (Laverov et al., 2004; Yarmolyuk, personal communication, 2013, etc.) midalkaline - alkaline rocks including known Pleistocene carbonatites of Hanneshin, Afghanistan and, also, of one of Arabia are subordinated (2 group of rocks). Rocks of 1 and 2 groups are interpreted by us as a tectonic-magmatic couple due to one from physics etc. In this case, at least, partial compensation of subduction compression by the intraplate extension is possible. Cenozoic intraplate rocks intraplate carbonatite-derived depth of the melt - an argument in favor of the African superplume influence on the magma plume of a large region, which is in agreement with effective tomography of the well-known J. Ritsema's team (Bull et al., 2009).

\section{OIL AND GAS, HYDROCARBONS (HC), SOME NOTES}

There are known materials about of Caspian Sea OIL / HC resources or productivity decreasing in north - northeast (N - NE) tectonic direction or lineament as stressed by known Prof. V. Khain with 
co-authors in the Explanatory map of Caspian Sea region scale 1:2 500000 etc. (Khain, 2001; Leonov et al., 2010). This decreasing is as follows: from extremely rich Persian Gulf to South - Middle North Caspian Sea. It is in agreement with the increasing distance from the African superplume by effective tomography (Bull et al., 2010), tectonics etc.

More specifically, this HC super belt is as Persian Gulf - Russian Arctic coast, due to old Russian HC maps, ex., USSR oil structures map scale 1:2 500000 etc. Also, important as HC traps salt domes in the east Persian Gulf are oriented due to this tectonic direction. Relation of HC field with faults is obvious. Other possibility for $\mathrm{HC}$ fields is combining of well-known $\mathrm{H} 2$ fluxes from the depth on faults with organic $\mathrm{C}$ in sediments.

Some experienced $\mathrm{HC}$ specialists believe that there are no strong contradictions in combined biogenic and abiogenic data now. HC fields are only of Cenozoic age, maybe the very Quaternary age for oil and younger age for the gas (not Riphean - Paleozoic - Mesozoic fields) due to a high mobility of HC. ex. There are data about ca $1 \mathrm{~m} /$ year HC migration. A. Timurziev (Timurziev, 2007, 2015 etc.), S. Marakushev, 2015; R. Seyful-Mulyukov, 2013 etc. think about the very deep abiogenic HC versus biogenic ones. In this context materials on mud-volcano-like structures on Mars comparing to known muds of Azerbaijan, Iran etc. by Skinner and Mazzini, 2009 etc. (fig.). It is well-known that mudvolcanoes or simply muds directly deal with $\mathrm{HC}$ fields.

No doubts about fault-related $\mathrm{HC}$ fluid input and also magmatic heat input sometimes. But biogenic factor is of great importance and main, surely. More data on HC peculiarities in the region studied needed.
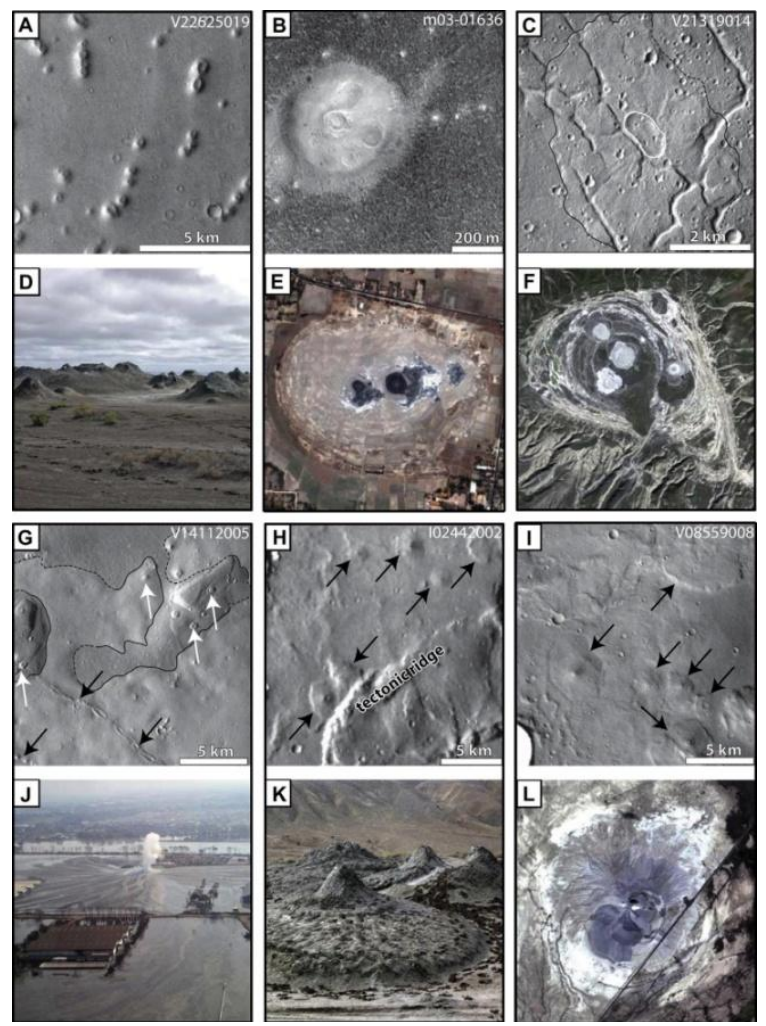

Fig15. Comparison of Azerbaijan, Iran (L), Java mud-volcanoes (muds) with mud-like structures of Mars after Skinner and Mazzini, 2009. Mud directly deals with OIL and other hydrocarbons.

\section{CONCLusions}

1. Some common geo-similarities on Cenozoic events in the region studied were revealed. At least, in East Iran important north-east tectonic-magmatic zoning and partly, metallogenic one (metallogeny under the leadership of known regional trio as E. Romanko, A. Houshmandzadeh, and M.A.A. Nogole-Sadat) due to African superplume activity exists here. It caused directly by known subduction of the Arabian plate under the Central Iran. African superplume activity strongly controls magmatism, "hot" regional tectonic regime, and, at least, partly - metallogeny in the region studied. Also, African superplume closely deals with known Jurassic Karoo flood basalts 
Some New and Previous Materials on Alpine Magmatism, Tectonics, Melt and Fluid Inclusions, and Metallogeny in Eastern Iran

event in Africa, Paleogene magmatism in East Africa, since mainly Eocene (Pg2) subduction in Iran - Turkey etc., Neogene 11-9 Ma opening of Red Sea etc., and probably, delamination of a mantle lithosphere in East Mediterranean in Miocene and as a result - lack of regional $\mathrm{Cu}$ porphyry mineralization versus economic one in Eocene before important delamination.

2. Two different types of Cenozoic magmatic rocks exist in the region studied: 1 -intraplate alkaline and subalkaline rocks and 2 - shoshonite - latite series rocks and calc-alkaline ones mainly dealing with subduction - collision events. Low crystallization temperature $-690 \mathrm{oC}$ and High $\mathrm{H} 2 \mathrm{O}$ content up to $6 \mathrm{wt}$. \%, and natural melt chilling were revealed for a probably Quaternary subduction-related rhyolite of the Bazman volcano (all data on melt inclusions led by V. Prokofiev) Sudden high/very high crystallization temperature, up to $1220 \mathrm{oC}$ on melt inclusions in High-K probably subduction-related dacite of remnant subduction were received too. Otherwise, for intraplate rocks as well for shoshonite - latite subduction related ones melt inclusions are not typical due to proposed warm conditions.

3. Eocene (Pg2) subduction-related shoshonite - latite series rocks almost in the whole region are characterized by an economic $\mathrm{Cu}-\mathrm{Au}$ mineralization with a subordinate different mineralization $(\mathrm{Cu}-$ $\mathrm{Pb}-\mathrm{Zn}-\mathrm{Au}-\mathrm{Ag}, \mathrm{Hg}-\mathrm{As}$, Au-Ag low-sulphide, Ag-sulfide with $\mathrm{Au}$ (?) etc.). Cu mineralization deals with deep basic enriched water-containing source. $\mathrm{Cu}$ mineralization disappears with time and higher in general magmatic section after disappears of relation with deep enriched source using (Haschke et al., 2010). Intraplate rocks bear, at least, REE, $\mathrm{P}$, also $\mathrm{Sr}, \mathrm{Ba}$, Th, and U mineralization.

\section{ACKNOWLEDGMENTS}

We are extremely grateful to outstanding regional trio - E. Romanko $\dagger$, A. Houshmandzadeh, and M.A.A. Nogole-Sadat $f$ for their leadership in the field works and comprehensive care; also - to famous V.E. Khain $\dagger$, M. Goncharov $\dagger$, and N. Bozhko (consulting, discussion, advices); V. Trifonov, V. Burtman, I. Pospelov, M. Kopp, E. Leven and V. Volkov; Yu. Leonov†, M. Leonov, M. Fedonkin, M. Khutorskoy, B. Polyak and V. Lavrushin; V. Yarmolyuk, D. Alexeev, M. Pevzner, M. Buyakayte, D. Astafiev, M. Rodkin, I. Gablina, Yu. Malinovsky† , S. Palandzhyan and G. Nekrasov; A. Knipper†, S, Ruzhentsev†, V. Kovalenker, V. Rusinov†, A. Girnis, A. Mokhov, N. Tarasov, S. Bubnov and A. Dockuchaev; G. Moralev, G. Gogonenkov et al., N. Panasuk†, also to A. Meskhi $\uparrow$ from Kazan, Tatarstan; M. Hosseini, M. Ziaii, and more other geo-specialists for long-term discussion, consulting analytical help etc.

The research was supported by the State program no. 0135-2018-0034 (Geological Institute, Russian Academy of Sciences; N. Chamov, and partly supported by the Russian Foundation for Basic Research, project no. 17-05-0000).

\section{REFERENCES}

[1] Abdullah S., Chmyrev V., Dronov V., Ed., 1980. Geology and Mineral Resources of Afghanistan. Moscow: Nedra. Book 1, p. 535, Book 2, 336 p. (in Russian).

[2] Alinaghi A., Koulakov I., Thybo H., 2007. Seismic tomographic imaging of $P$ - and $S$-waves velocity perturbations in the upper mantle beneath Iran. Geophysical J. International, 169, pp. 1089-1102.

[3] Artemiev M., 1971. Some features of the deep structure of the Mediterranean type basins according to isostatic gravity anomalies. Bull. Moscow Society of Naturalists, Dept. Geol., N 4, pp. $30-41$ (in Russian).

[4] Bogatikov O.A, Kovalenko V.I., Yarmolyuk V.V., Bubnov S.N., 1987. Series of igneous rocks: problems and solutions. Reports of the Academy of Sciences of the USSR, Series Geol, N 3, pp. 3-12 (in Russian).

[5] Bortnikov N.S., Prokofyev V.Yu., 2007. Major mesothermal gold ore deposit of Russia: composition and origin of ore-forming fluids. Digging Deeper. Proceedings of the Ninth Biennial meeting of the society for geology applied to mineral deposits. Dublin, Ireland. 2007. Dublin Published Irish Association for Economic Geology, pp. 793-796.

[6] Bortnikov N.S., Vikentiev I.V., 2004. Modern ore minerals formation in the oceans. Ore deposits and oreforming processes.M. IGEM, pp. 325-356. In Russian.

[7] Bull A.L., McNamara A.K., Ritsema J., 2009. Plume clusters and thermochemical piles. Earth Planet. Sci. Lett. Vol. 278, pp. 152-162.

[8] Burtman V.S., 2012. Tien-Shan and High Asia. Geological Institute RAS Transactions. M: GEOS, 188 p. In Russian. 
Some New and Previous Materials on Alpine Magmatism, Tectonics, Melt and Fluid Inclusions, and Metallogeny in Eastern Iran

[9] Dilek Y., Imamverdiyev N.A., Altunkaynak S., 2010. Geochemistry and tectonics of Cenozoic volcanism in the Lesser Caucasus (Azerbaijan) and the Peri-Arabian region: Collision-induced mantle dynamics and its magmatic fingerprint. International Geology Review, vol. 52, issue 4-6, pp. 536-578.

[10] Haschke M., Ahmadian J., Murata M., McDonald I., 2010. Copper mineralization prevented by arc-root delamination during Alpine-Himalayan collision in Central Iran. Economic Geologists, v. 104, pp. 885895.

[11] Hushmand-Zadeh A., Nabavi M.H., 1986. Metamorphic Map of Iran Scale 1: 2,500,000. Tehran: Geol. Survey. Iran.

[12] Imamverdiyev N.A. 2000. Geochemistry of Late Cenozoic volcanic complexes Lesser Caucasus Baku. Nafta-Press. 2000. 192 p.

[13] Imamverdiyev N.A. 2008. Delamination of subducted lithospheric slab as the cause of Late Cenozoic volcanism in the Lesser Caucasus. News of State Baku University. Natural sciences Series. no 3, pp.536578.

[14] Khain V., 2001. Tectonics of Continents and Oceans (year 2000). Moscow: Scientific World, 2001. 606 p. In Russian.

[15] Khain V., Goncharov. M., 2006. Endogenic processes of different tectonic settings. Herald of MSU. Vol. 5. pp. 15-23. In Russian.

[16] Knipper A., Dobretsov N., Bogdanov N., 1992. Ophiolites and "orogenic lherzolites" of Bet Cordillera, Spain. Reports of the Academy of Sciences. Geological Series. N 12, pp. 8-24. In Russian.

[17] Koronovsky N.V., Demina L.I., 1999. Collisional stage of the Caucasian sector Alpine fold belt: geodynamics and Magmatism. Geotektonika, no 2, pp. 17-35. In Russian.

[18] Koronovsky N.V, Lomize M.G., 2006. Tectonic accretion and collision as the steps of forming intracontinental fold belts. Areas of active tectonics in modern and ancient history of the Earth. Materials of 39 Tectonic meeting, vol. 1, Moscow: GEOS. pp. 353-357. In Russian.

[19] Laverov N., Kovalenko V., Yarmolyuk V. et al., 2006. The latest volcanic activity in Northern Eurasia: Zoning and environment of formation. Russian Academy of Sciences Reports. vol. 410. no 4, pp. 498-502. In Russian.

[20] Leonov Yu. G., Edit., 2007. Alpine history of the Great Caucasus. Moscow: GEOS. 368 p. In Russian

[21] Leonov Yu., Volozh Yu., Antipov M. et al., 2010. Consolidated crust of Caspian region. M. GEOS. 64 p. In Russian.

[22] Luchitsky I.V., 1985. Palaeovolcanology. Moscow: Science (Nauka), 235 p. In Russian.

[23] Marakushev A.A., 1988. Petrology. Moscow. MSU publishing, 205 p. In Russian.

[24] Milanovsky E.E., Koronovsky N.V., 1973. Orogenic volcanism and tectonics of the Alpine belt of Eurasia. Moscow: Nedra, 280 p. In Russian.

[25] Nogole-Sadat, M.A., 1985. Les Zones de Decrochement et les Virgation Structurales en Iran: Geol. Survey of Iran, Report, 55, 259 p.

[26] Pars Kani Co, 2003. Preliminary report on the prospection of epithermal gold mineralization in Chahnali north of Bazman, Sistan-va-Baluchestan province, Southeast Iran. International Report. 136 (in Farsi language with English abstract).

[27] Pospelov I., 2009. Tectonics of Pamir. Tectonics and geology. V. 5, pp. 15-25.

[28] Prokofiev V.Yu., 2000. Geochemistry of ore-forming fluids of hydrothermal gold deposits of different genetic types according to the study of fluid inclusions. Moscow. Science (Nauka), Novosibirsk, 192 p. In Russian.

[29] Prokofiev V.Yu., Bortnikov N.S., Zorina L.D. et al., 2000. The Darasun intrusive-related gold-base metal deposit, Eastern Transbaikalia, Russia: Petrochemical, melt and fluid inclusion, REE and stable isotope (O, C, and S) studies. Applied Mineralogy. Rammlmair et al., Eds. Balkema, Rottertdam, Brookfield, 399-402 pp.

[30] Romanko E., Kokorin Y.., Krivyakin B. et al., 1984. Outline of metallogeny of Anarak Area (Central Iran). Ministry of mines and metals. Geological Survey of Iran. Rep. 21, Tehran. Technoexport, 136 p.

[31] Romanko E., Voinkov D., Houshmandzadeh A. et al., 2000. Characteristic features of ore mineralization in the Sistan-Baluchestan Province, SE Iran: evidence from fluid inclusions data. International conference on geology of Mediterranean belt. Absracts. Beograd.

[32] Romanko E., Houshmandzadeh A., Nogol-Sadat M.A.A. et al., 2000. Metallogenic zonation in Seistan and Baluchestan Province, East Iran. Transactions (Doklady) of Russian Academy of Sciences. V 370, N 1, pp. 76-79, in Russian. 
[33] Romanko E.F, Romanko A.E., Meskhi A.M., 1998. Geology and Geochemistry of Mesozoic-Cenozoic Magmatic Formations in Northeastern Algeria. Transactions of the Russian Academy of Sciences, V 362, N 7, pp. 925-927, in Russian.

[34] Romanko A., 2005. New data on Cenozoic subalkaline intraplate rocks the East Iran. Reports of the Russian Academy of Sciences. Earth Sciences Section. V 404, N 7, pp. 510-513.

[35] Romanko A., Imamverdiyev N.A., Prokofiev V., Vikentiev I., Savichev A., Stepanov S., 2013. Alpine tectonic-magmatic-metallogenic peculiarities in West Baluchestan, Middle East: new data, "hot" tectonics, inclusions, discussion, hydrocarbons (oil, HC), and constraints. Universal Journal of Geoscience, V 1, pp. 130-138.

[36] Romanko A., Imamverdiyev N. A., Prokofiev V. et al. 2012. On Cretaceous - Quaternary tectonic magmatic - metallogenic peculiarities in East Iran and South Tajikistan: new data melt inclusions, constraints, and some problems. European Seismological Commission Assembly. ESC. Book of abstracts. M. Polygraph, pp. 154-155.

[37] Rusinov V.L., 1985. Propilites and propilitization. M.: Nauka. 185 p. In Russian

[38] Sandwall E., Turkell N., Zor E. et al., 2003. Shear wave splitting a young continent collision. Geophys. Res. Lett., 30. no 24 b, pp.185.-194.

[39] Shilov V.N, 1997. Comparison of Neogen-Quaternary volcanism on Sakhalin and East Sikhote-Alin. Volcanology and Seismology. V. 18. pp. 517-528.

[40] Timurziev A.I., 2007. On a creating of new oil-gas geology paradigm based on deep-filtration model. Geophysics. N 4. pp 49-60. In Russian.

[41] Trifonov V.G., Ruzhentsev S.V., 1984. Tectonic napes in East Pamir, Tajikistan and tectonic layering. Episodes. 32 IGC. Special Issue. pp. 15-20.

[42] Vikentiev I.V., Yudovskaya M.A., Mokhov A.V. et al. 2004. Gold and PGE in massive sulphide ores of the Uzelginsk deposit, Southern Urals, Russia. Canadian Mineralogist. V 42, N 5, pp. 651-665.

[43] Yarmolyuk V.V., Kovalenko V.I., 2003. Deep geodynamics, mantle plumes and their role in Central-Asian mobile belt formation. Petrology. V 11. N 6. pp. 556-586. In Russian.

[44] Yarmolyuk V.V., Kovalenko V.I., Bogatikov O.A., 1990. South Baikal "hot spot" mantle and its role in the formation of the Baikal Rift region. Reports of Russian Academy of Sciences. V 312. N 1. pp. 187191. In Russian.

\section{AUTHORS' BIOGRAPHY}

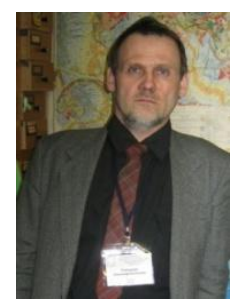

Alexander Romanko, Dr, Senior Researcher, Member of volcanology and chemistry of the Earth's Interior.International Association on

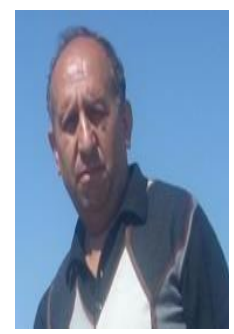

Nazim A, Imamverdiyev, Professor, Dr, well-known specialist in magmatism, metallogeny etc.

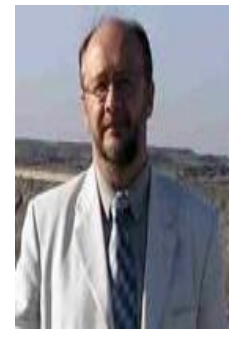

Vsevolod Yu, Prokofiev (Prokof 'ev), Professor, Dr, known specialist in Magmatism, metallogeny etc. 
Some New and Previous Materials on Alpine Magmatism, Tectonics, Melt and Fluid Inclusions, and Metallogeny in Eastern Iran

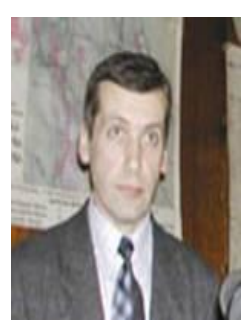

Ilya Vikentiev, Professor, Dr, known specialist in Magmatism, metallogeny, Urals, Altai etc.

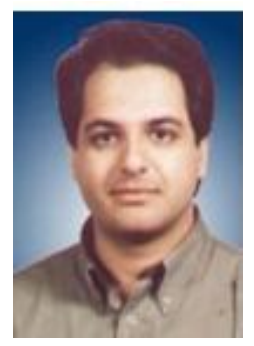

Bahman RASHIDI, senior geologist, specialist in metallogeny etc.

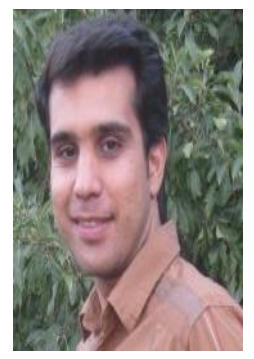

Alexander SAVICHEV, Dr., Professor, Laboratory Chief, known specialist in chemistry, environmental sciences, magmatism, metallogeny, etc.

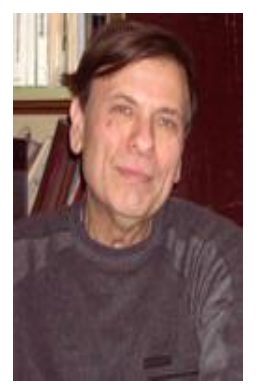

Mehrdad HEIDARI, senior geologist, specialist in metallogeny etc.

Citation: A. Romanko et.al. (2018). Some New and Previous Materials on Alpine Magmatism, Tectonics, Melt and Fluid Inclusions, and Metallogeny in Eastern Iran, International Journal of Mining Science (IJMS), 4(1), pp.11-28, DOI: http://dx.doi.org/ 10.20431/2454-9460.0401002.

Copyright: () 2018 A. Romanko et al.,. This is an open-access article distributed under the terms of the Creative Commons Attribution License, which permits unrestricted use, distribution, and reproduction in any medium, provided the original author and source are credited 